\title{
HYPERSPECTRAL RESOLUTION ENHANCEMENT USING MULTISENSOR IMAGE DATA
}

\author{
J. Bieniarz, D. Cerra, X. X. Zhu, R. Müller, P. Reinartz
}

German Aerospace Center (DLR), Remote Sensing Technology Institute (IMF), Oberpfaffenhofen, 82234 Wessling, Germany.

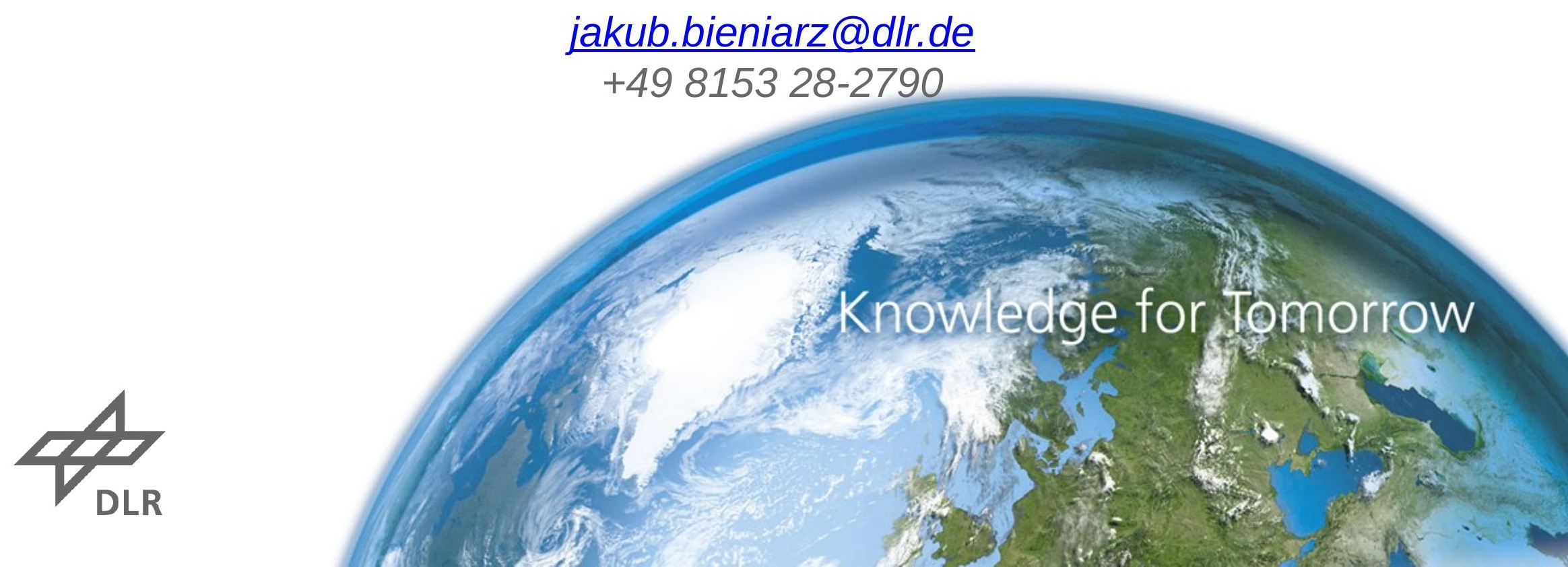




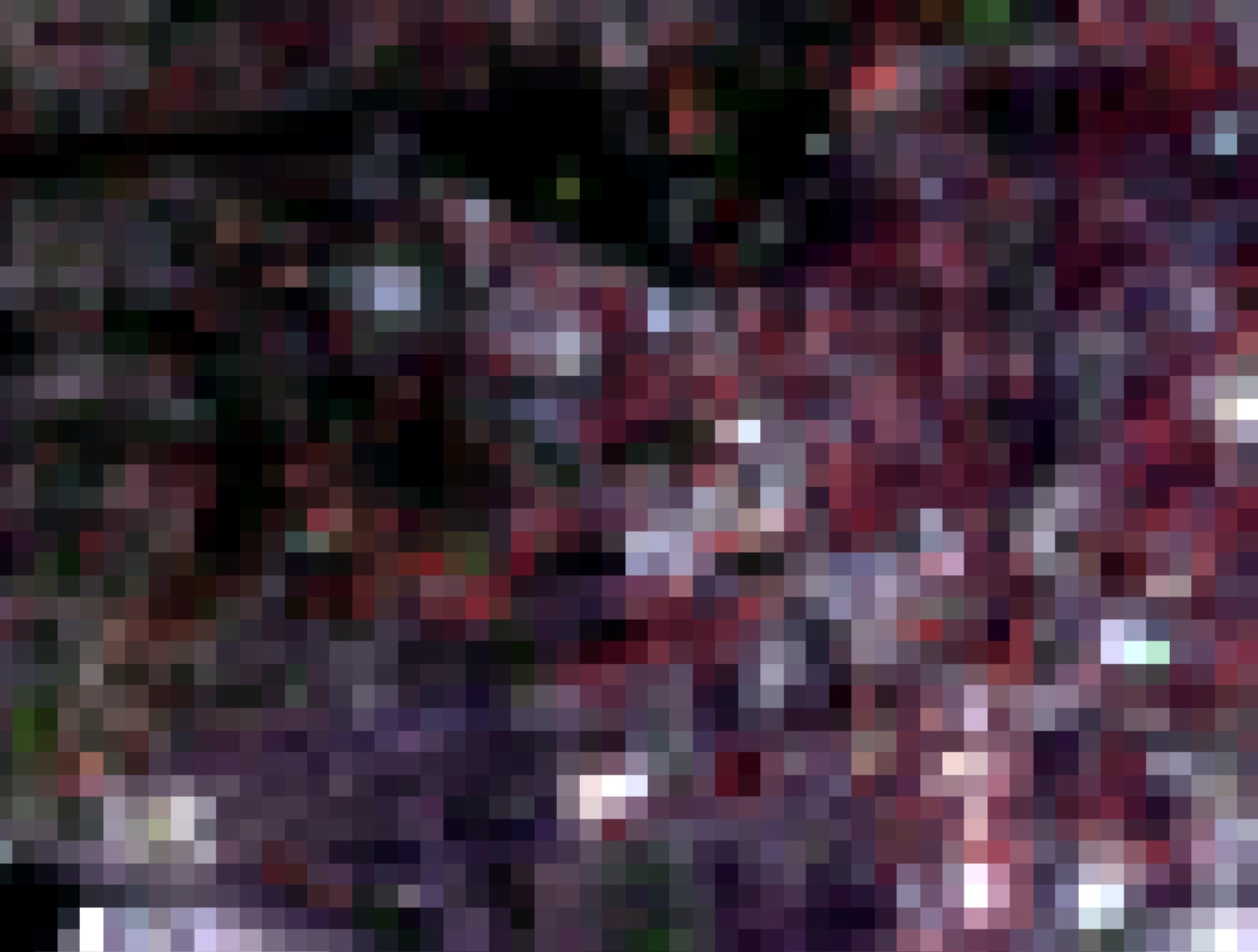




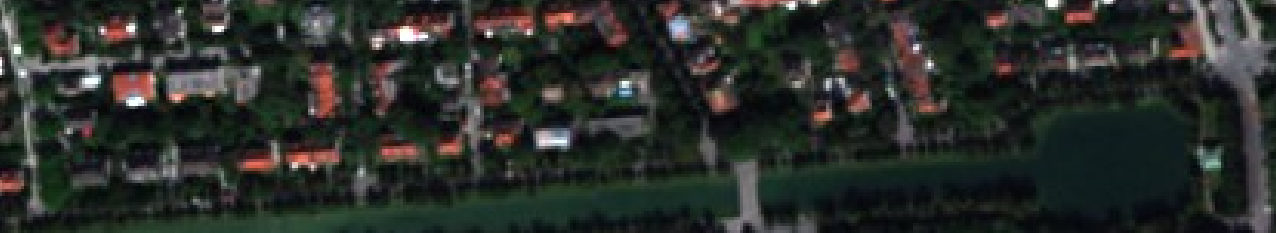

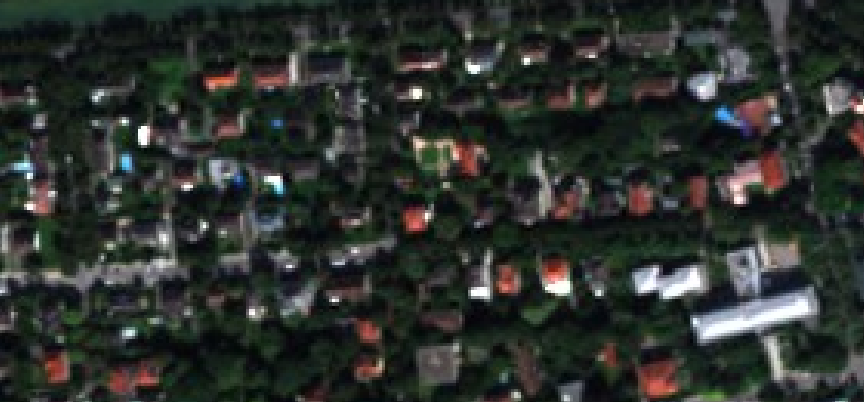

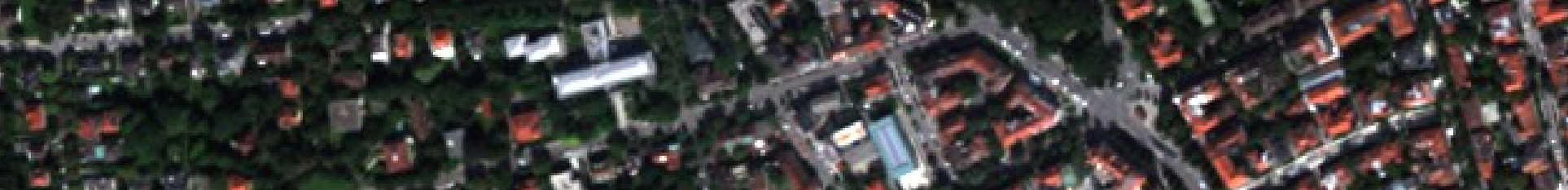

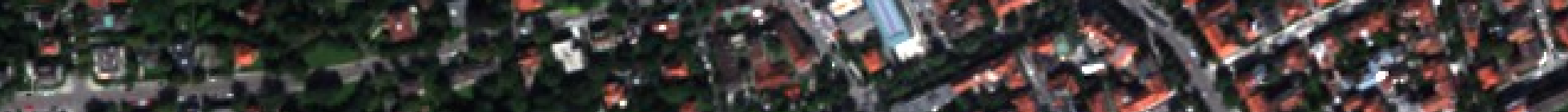

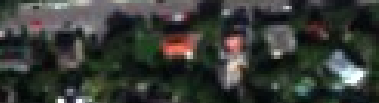

60

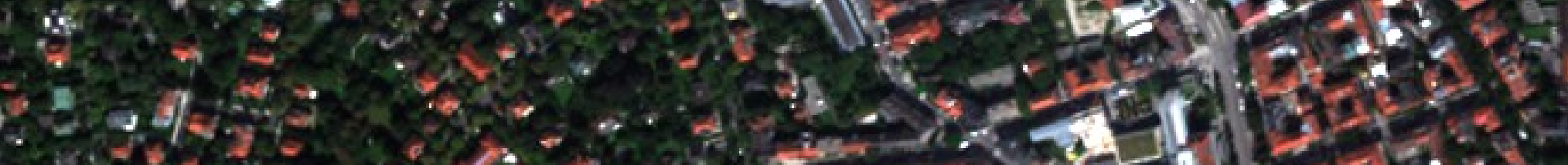

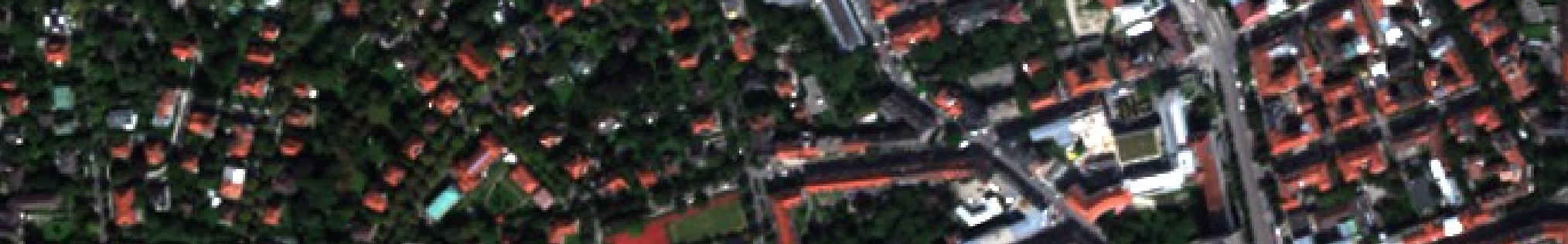

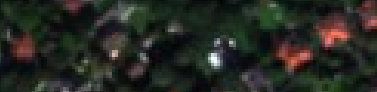

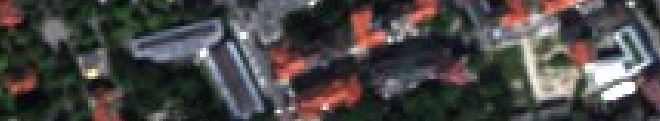

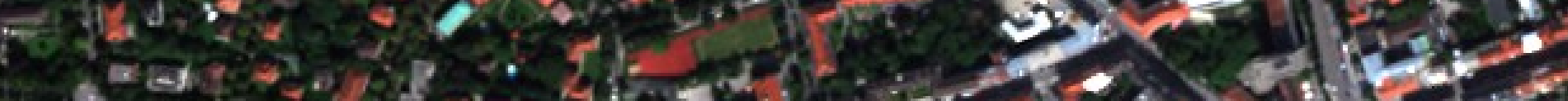

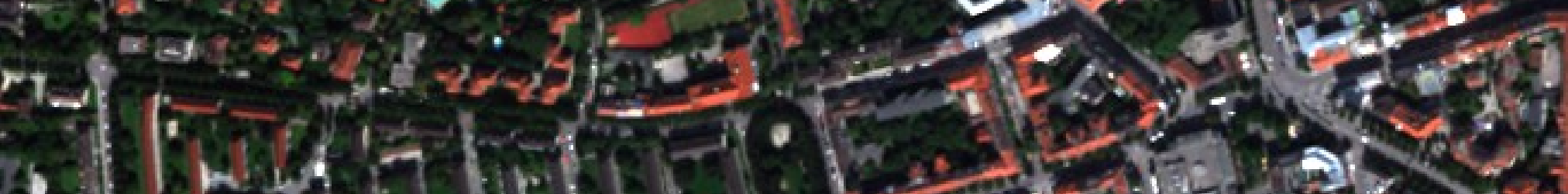

a

7

ind weris

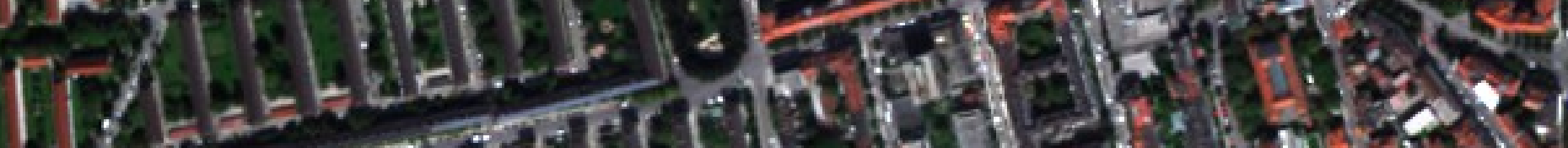

a.

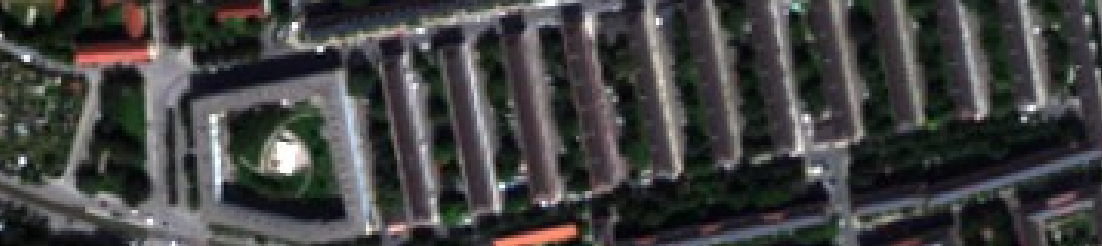
(2)

$E$

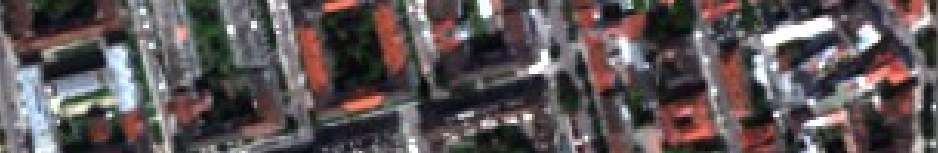

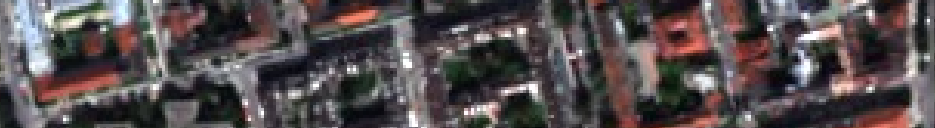
$=$

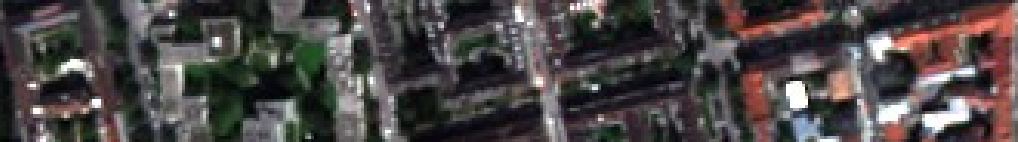

187.4 getos 


\section{Unmixing Based Resolution Enhancement}

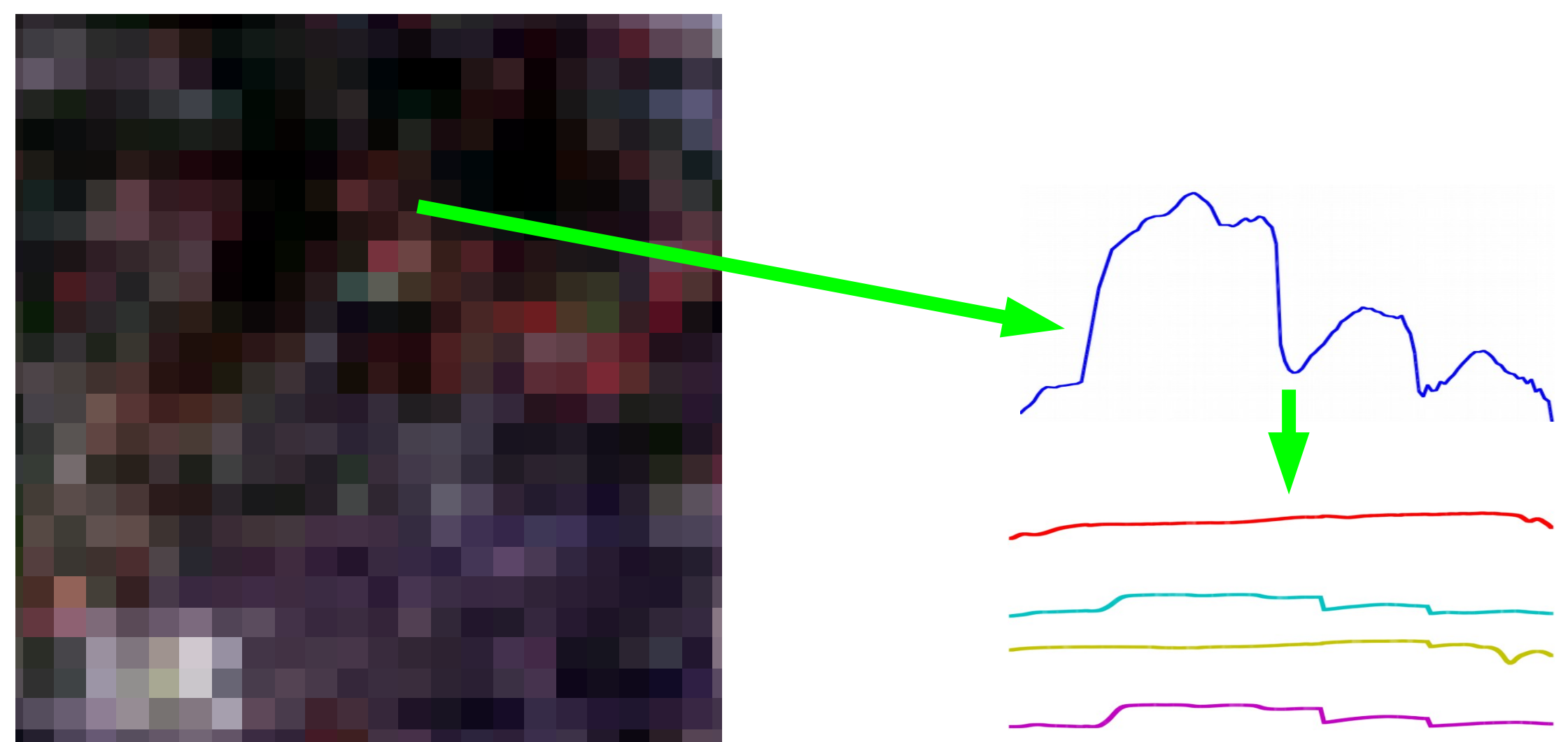




\section{Unmixing Based Resolution Enhancement}

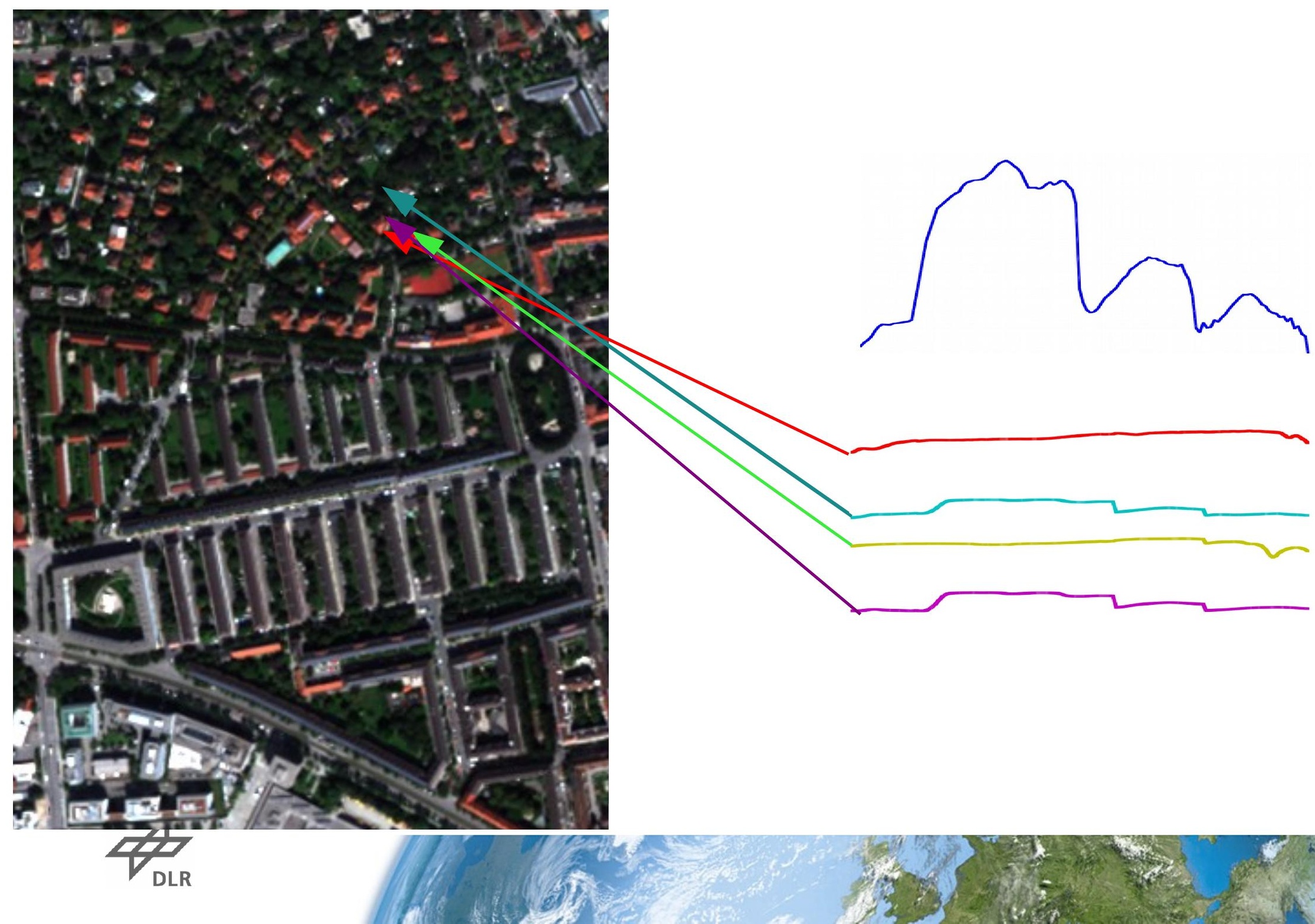


Highly Mixed Scenario

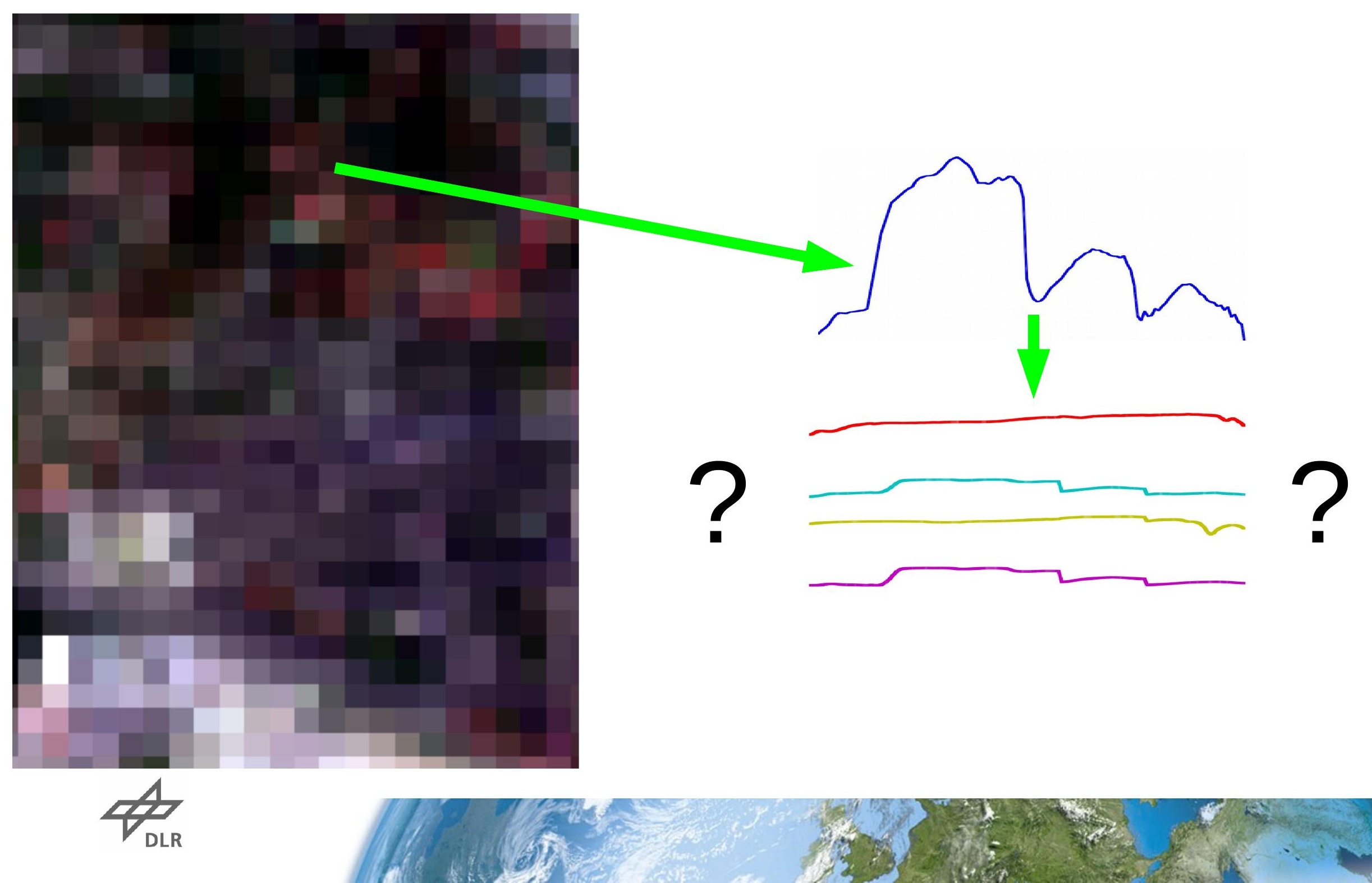


Multi-Look Joint Sparsity Fusion (MLJSF)

- Unmixing based image fusion

- Use of external (pre-defined) spectral libraries containing pure endmemebers

- Jointly sparse unmixing concept - spatial spectral imnixing 


\section{Sparse Spectral Unmixing - The Concept}

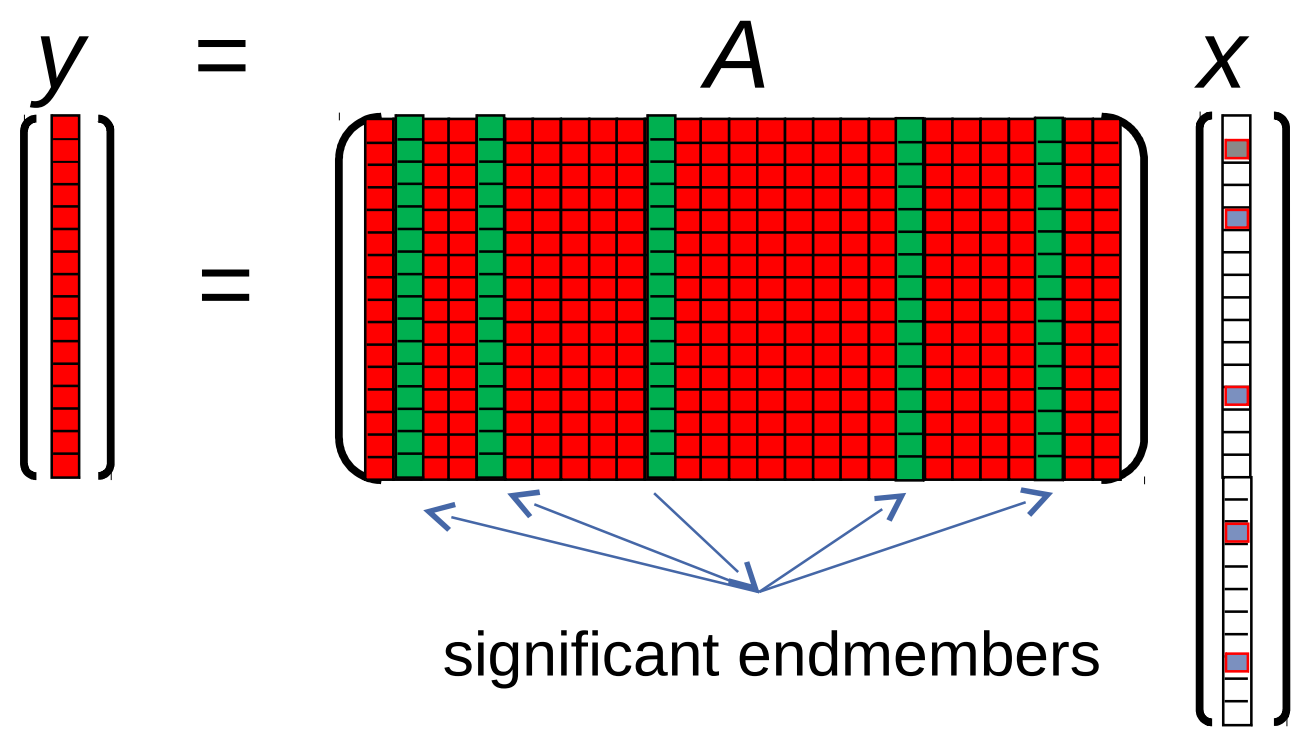

Use of large libraries

- Aster Spectral Library from JPL, USGS, JHU with 2463 spectra

- DLR Spectral Archive with 1609 spectra

Requirements:

- Higly accurate Atmospheric Correction

$$
-\min \|y-A x\|_{2}^{2}+\lambda\|x\|_{1} \text { s.t. } x \geq 0
$$




\section{Hyperspectral Image Resolution Enhancement Based on Jointly Sparse Spectral Unmixing}

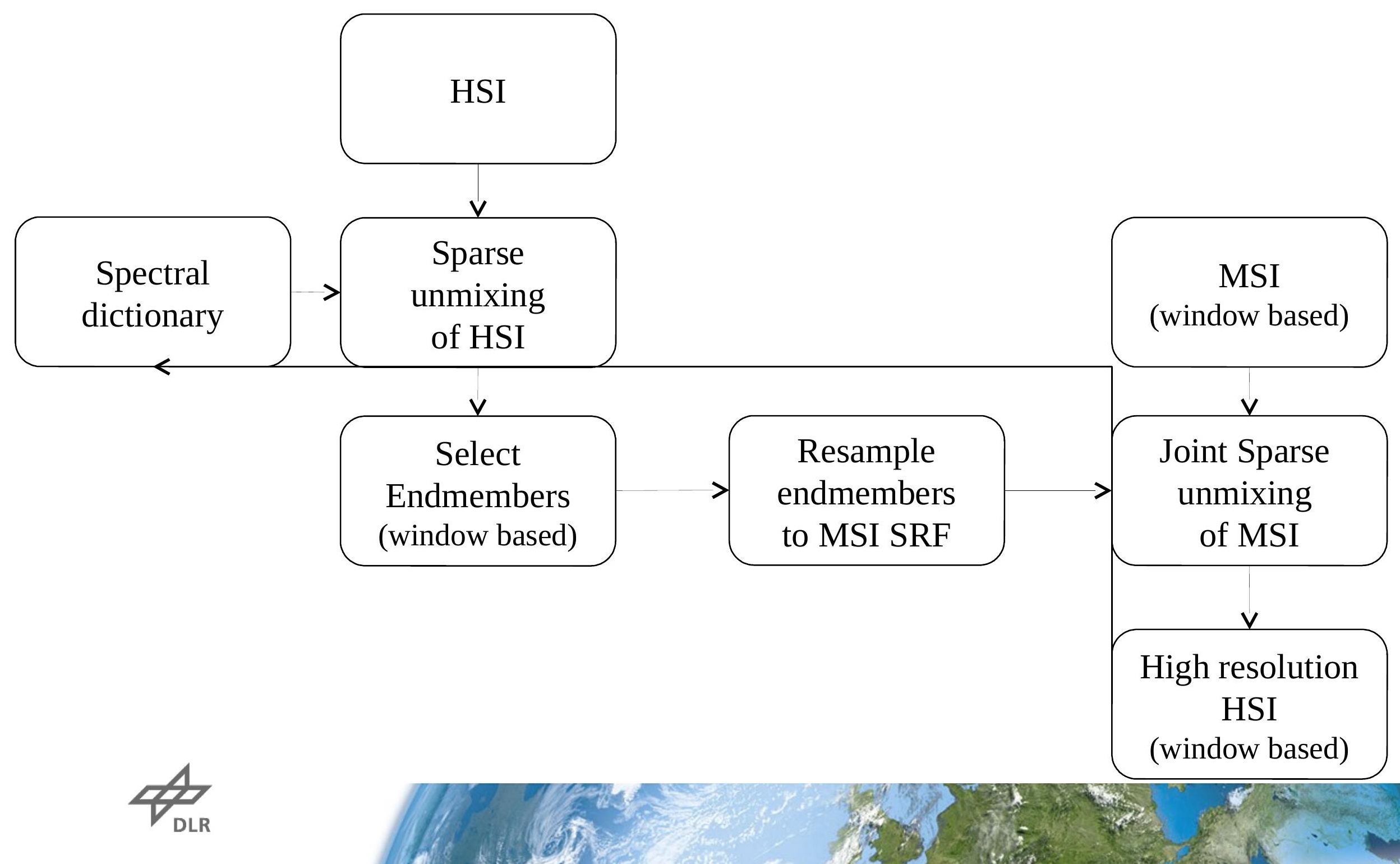




\section{Hyperspectral Image Resolution Enhancement Based on Jointly Sparse Spectral Unmixing}
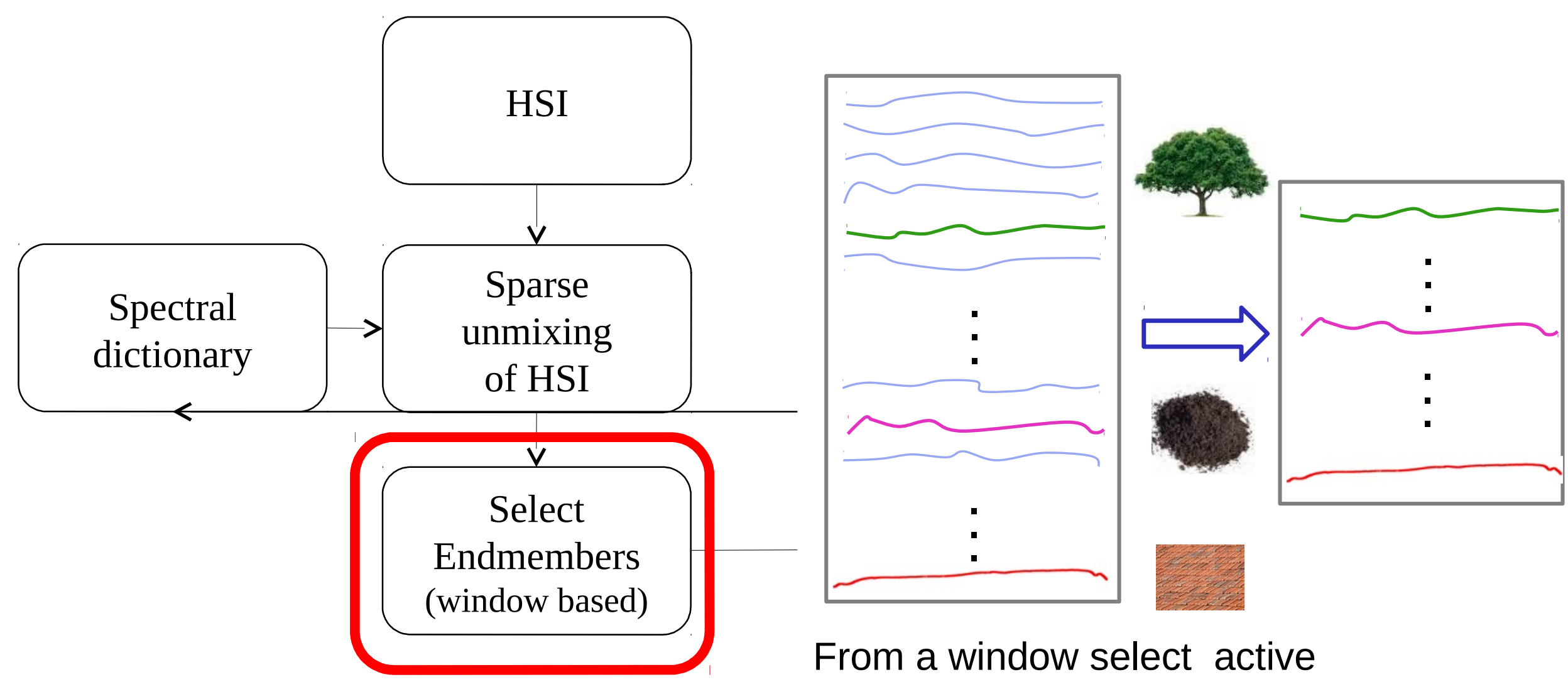

From a window select active endmembers and construct a new pruned dictionary for this region. 


\section{Hyperspectral Image Resolution Enhancement Based on Jointly Sparse Spectral Unmixing}

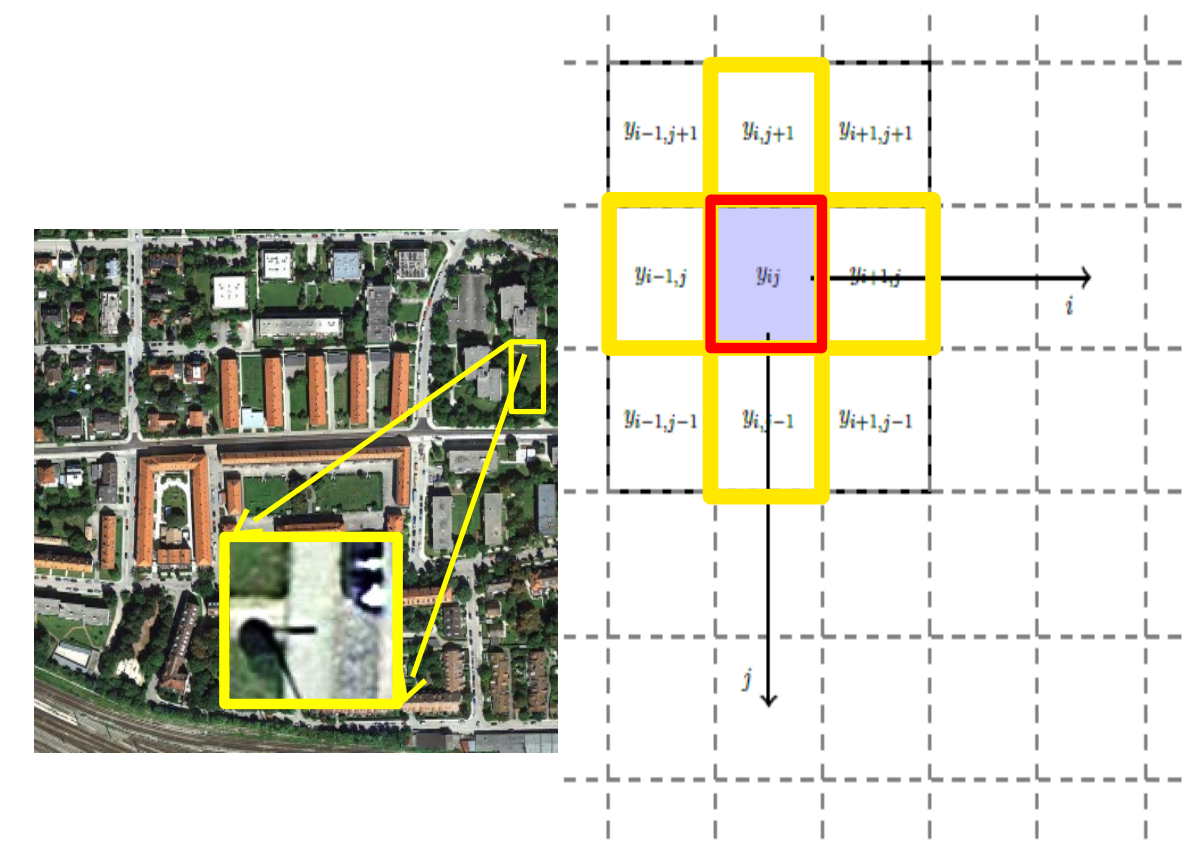

Unmix the window of the MSI using the Joinr Sparsity Model (JSM)

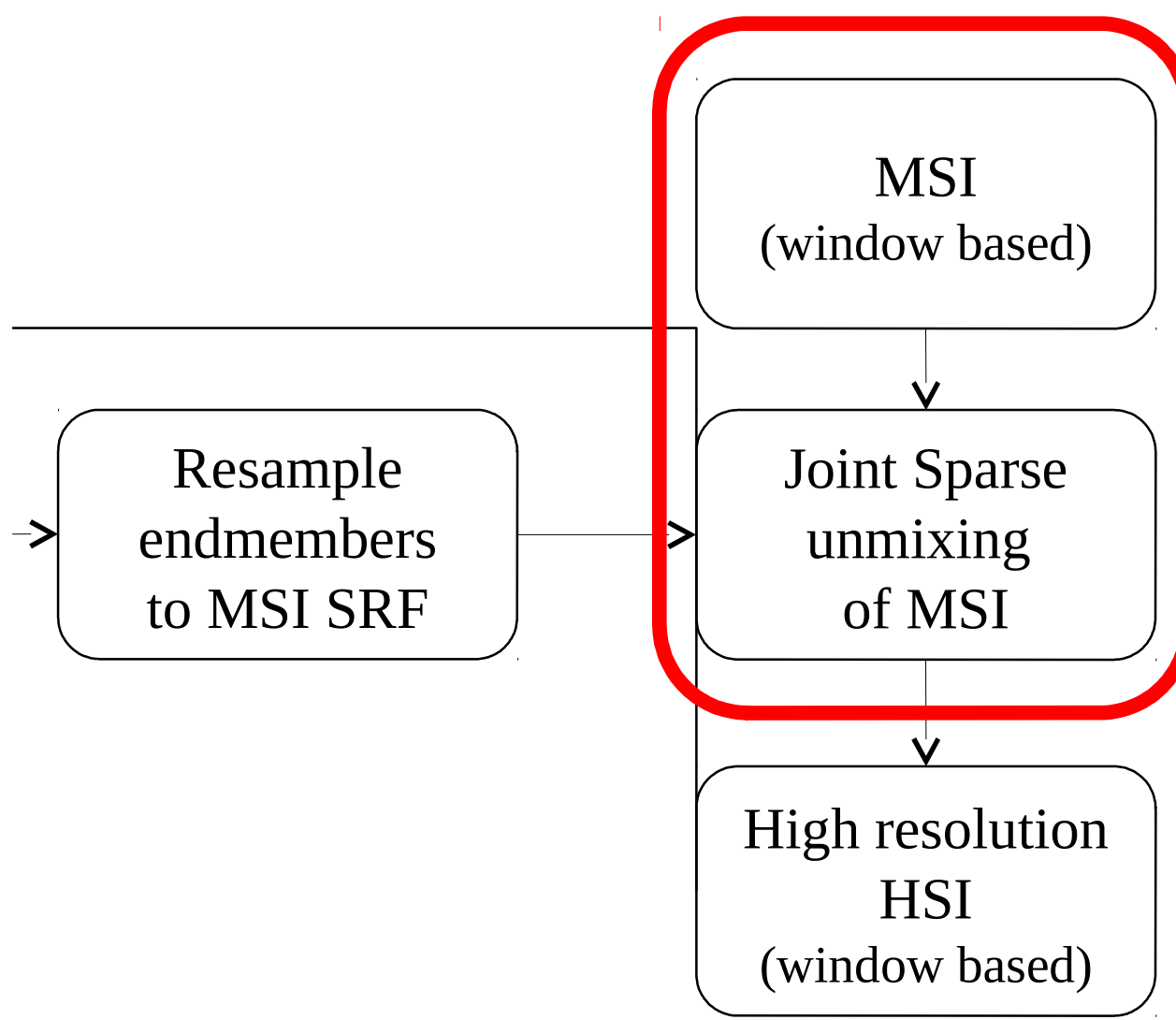




\section{EnMAP + World_View2}
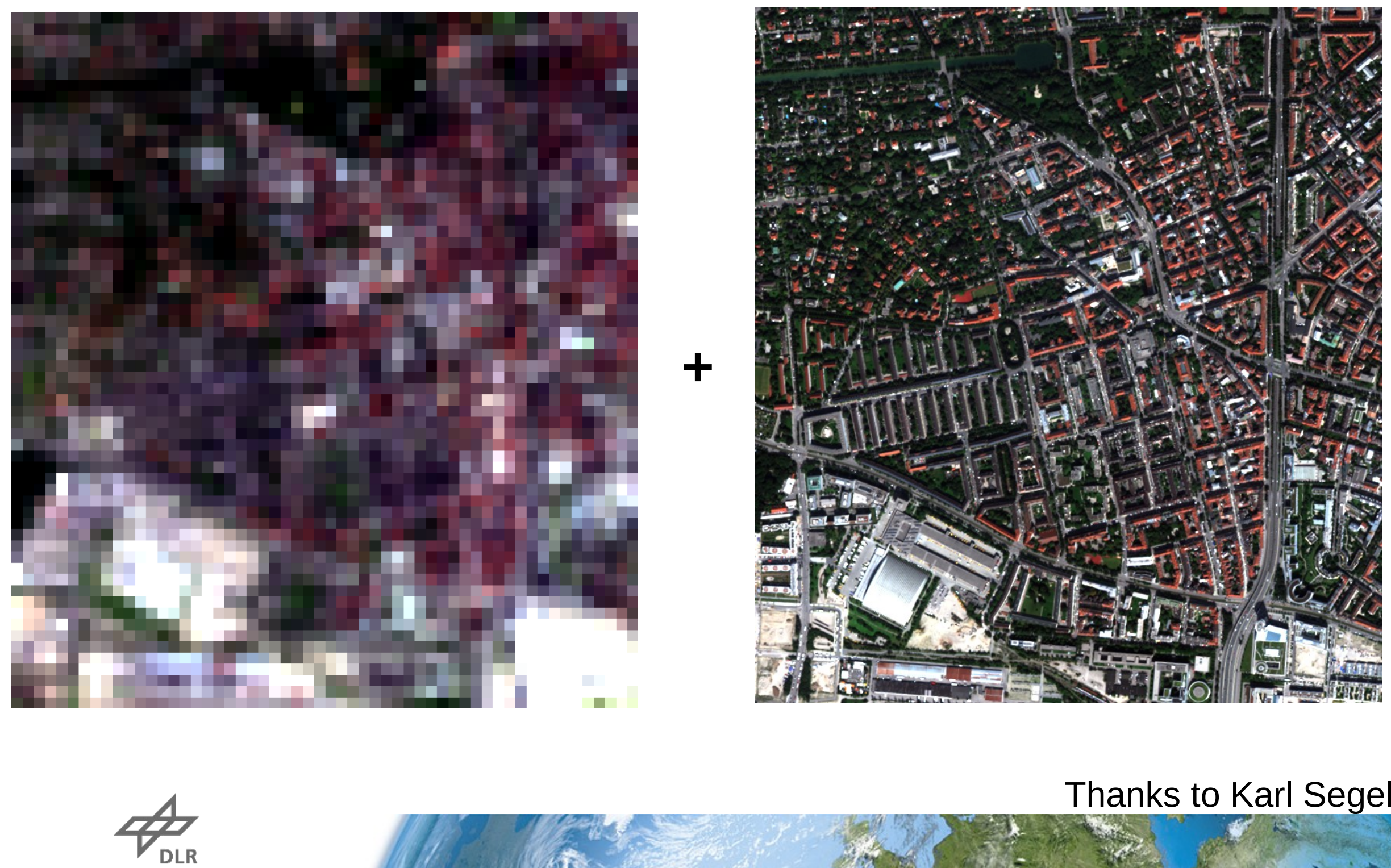

Thanks to Karl Segel 

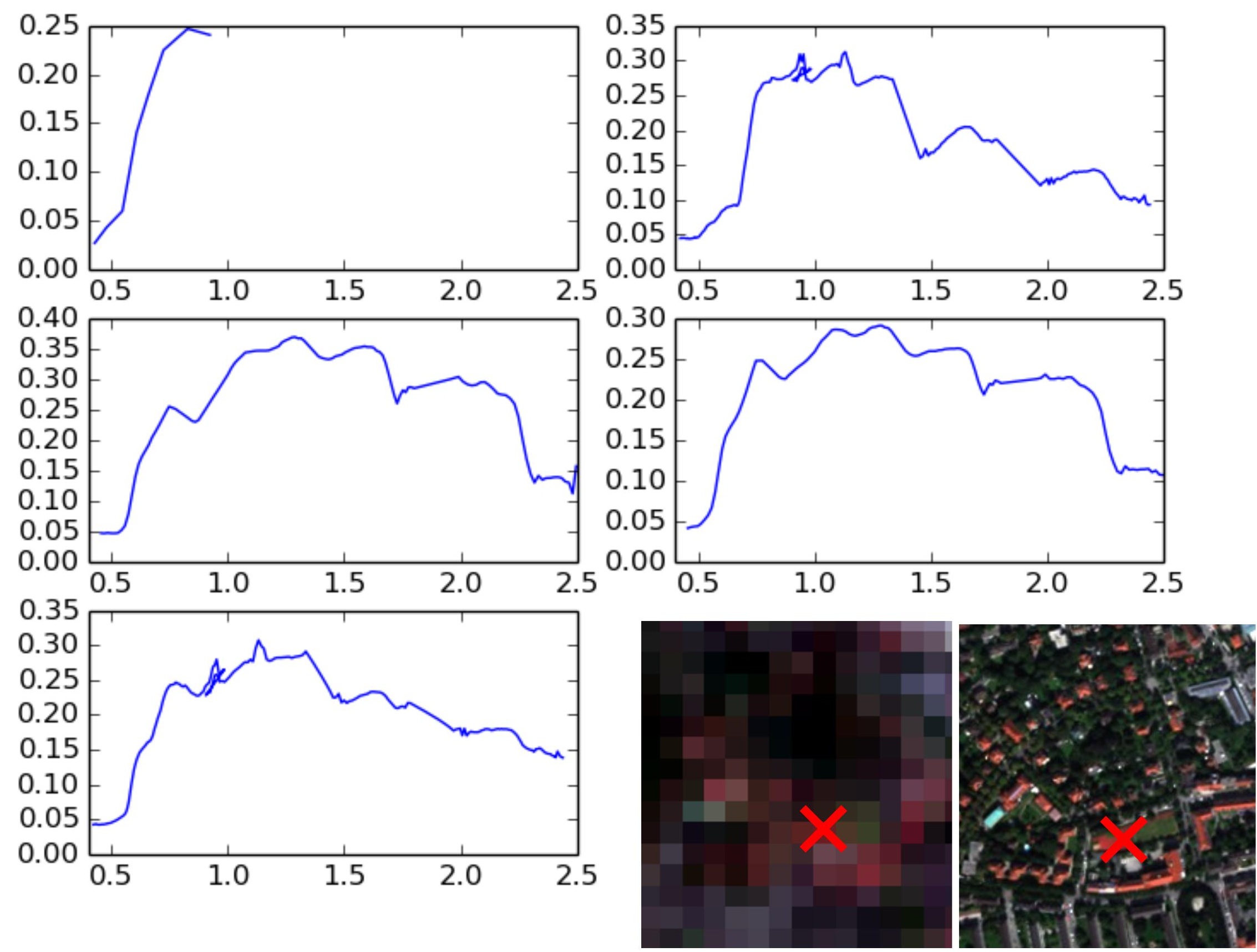

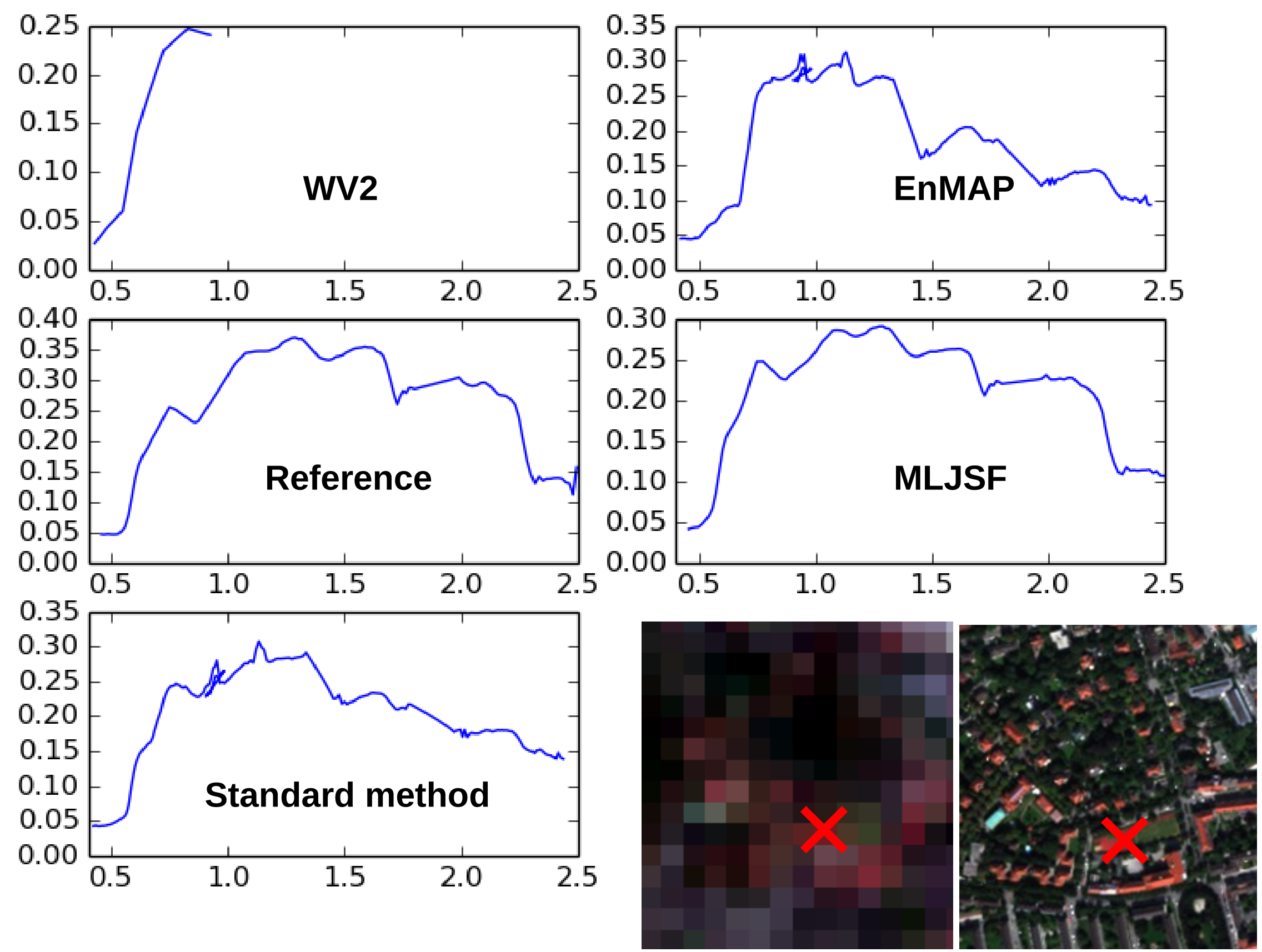

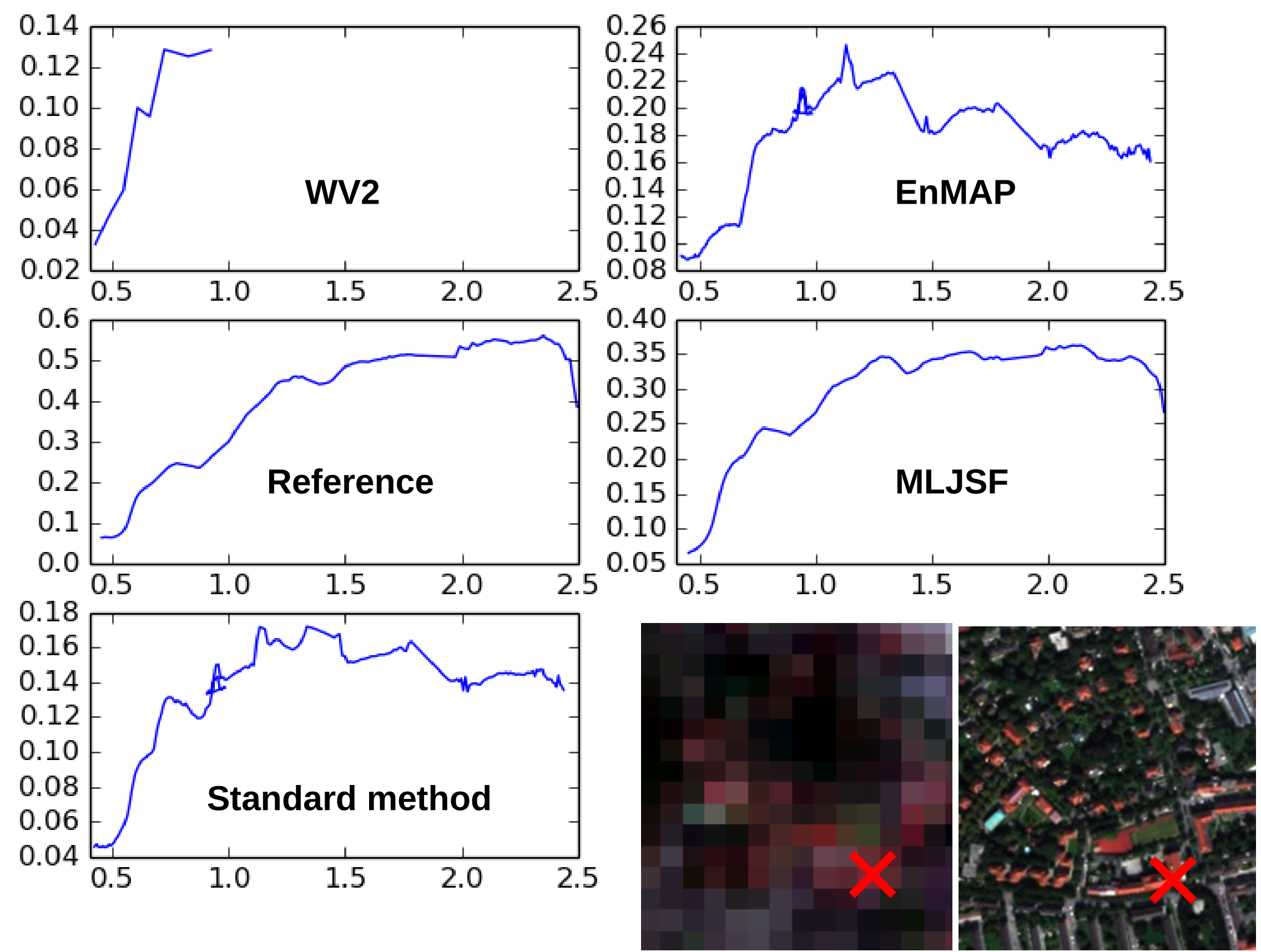


\section{Experiments - spatial resolution}

\section{HYPERION}

- 30m spatial resolution

- 220 spectral bands (152 after band reduction) - 10nm spectral resolution - 0.4-2.5 spectral range 


\section{Experiments - spatial resolution}

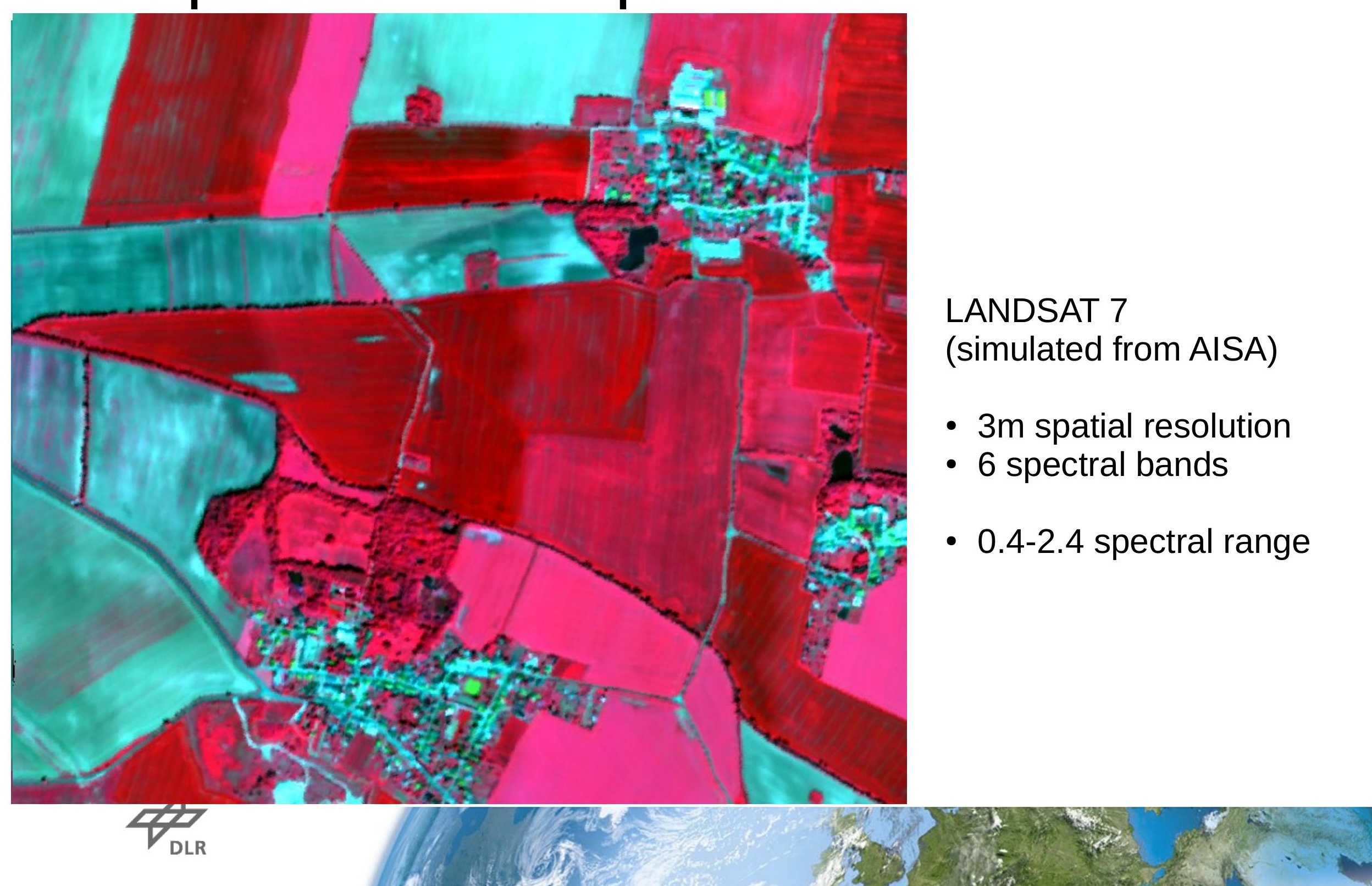




\section{Experiments - spectral resolution}

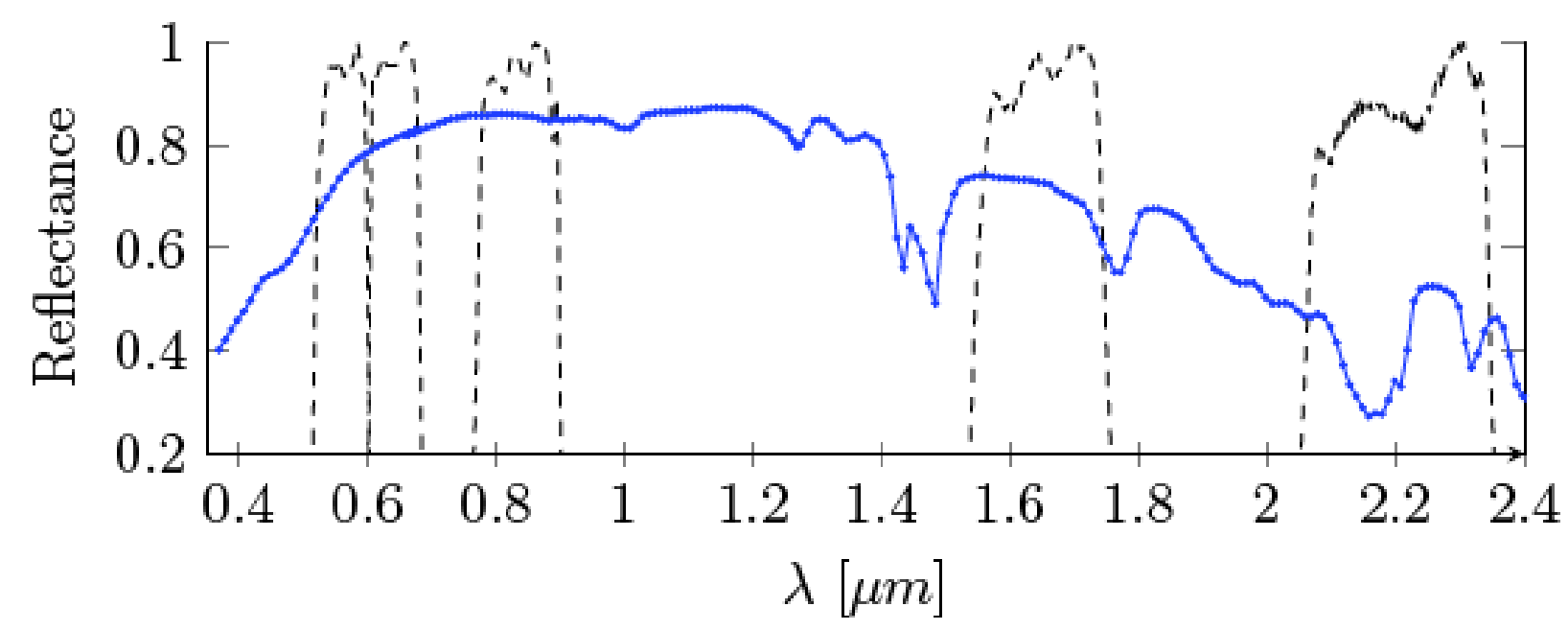

HYPERION

- 30m spatial resolution

- 220 spectral bands (152 after band reduction)

- 10nm spectral resolution

- 0.4-2.5 spectral range

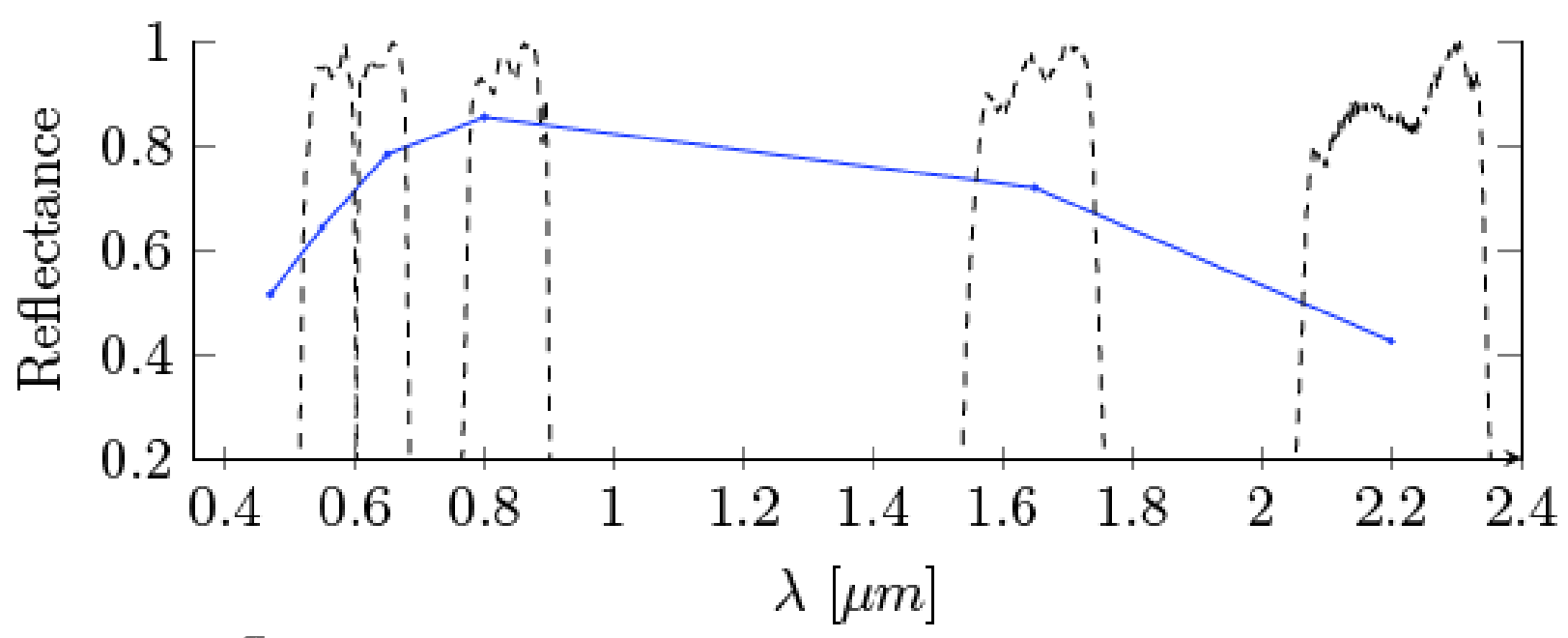

LANDSAT 7

(simulated from AISA)

- 3m spatial resolution

- 6 spectral bands

- 0.4-2.4 spectral range 


\section{Results}

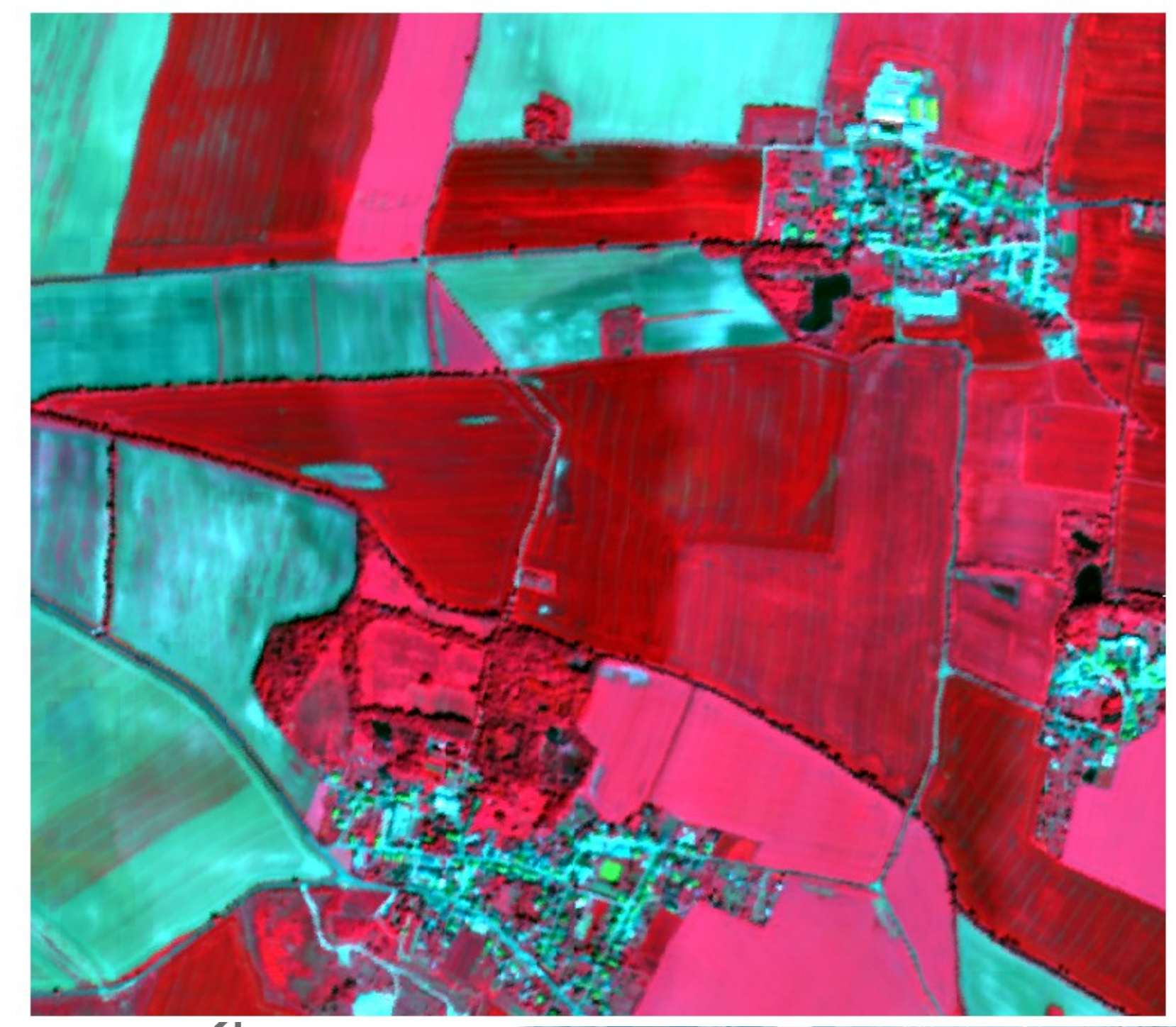

MLJSF

- 3m spatial resolution

- 152 spectral bands

- 10nm spectral resolution

- 0.4-2.5 spectral range

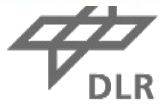




\section{Evaluation - 2 ways}

- Performance measure

- Quantitative comparison

- Application example

- SAM Classification 


\section{Quantitative analisys}

\begin{tabular}{l|l|l|l|l|l|} 
& SSIM & SADrad & NCC & NRMSE [\%] & Time [s] \\
\hline Hyperion & 0.16 & 0.121 & 0.82 & 91.6 & - \\
CNMF & 0.95 & 0.061 & 0.98 & 6.8 & 388.62 \\
MLJSF & 0.96 & 0.067 & 0.97 & 7.8 & 46.87
\end{tabular}

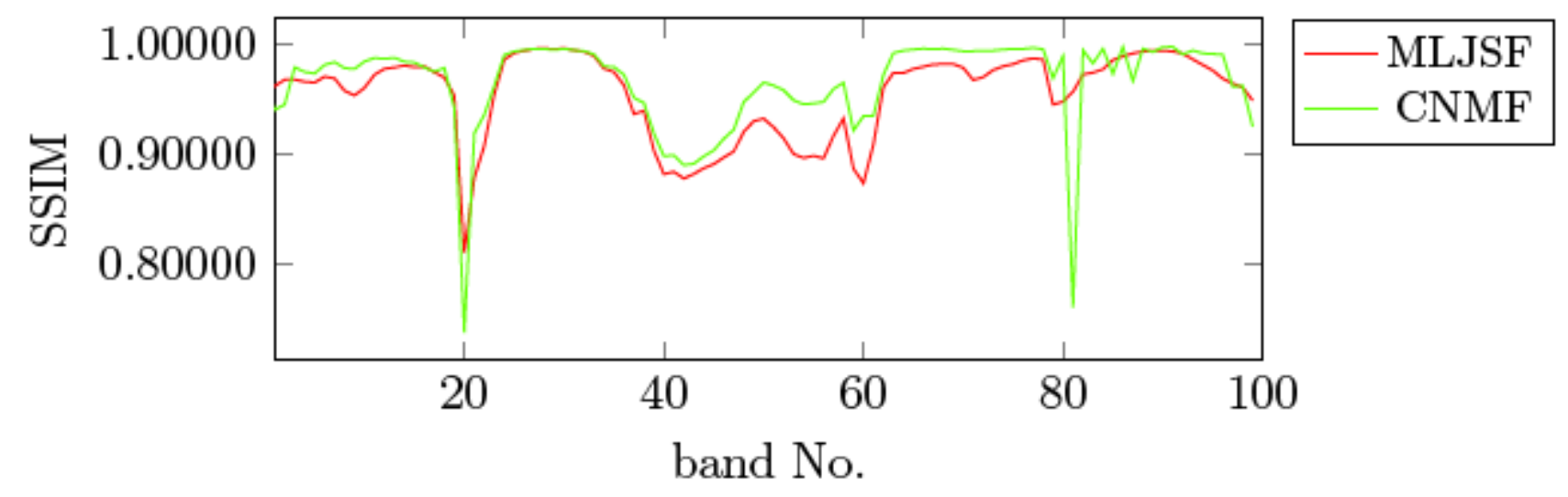




\section{Quantitative analisys}

\begin{tabular}{l|l|l|l|l|l|} 
& SSIM & SADrad & NCC & NRMSE [\%] & Time [s] \\
\hline Hyperion & 0.16 & 0.121 & 0.82 & 91.6 & - \\
\hline CNMF & 0.95 & 0.061 & 0.98 & 6.8 & 388.62 \\
MLJSF & 0.96 & 0.067 & 0.97 & 7.8 & 46.87 \\
\hline
\end{tabular}

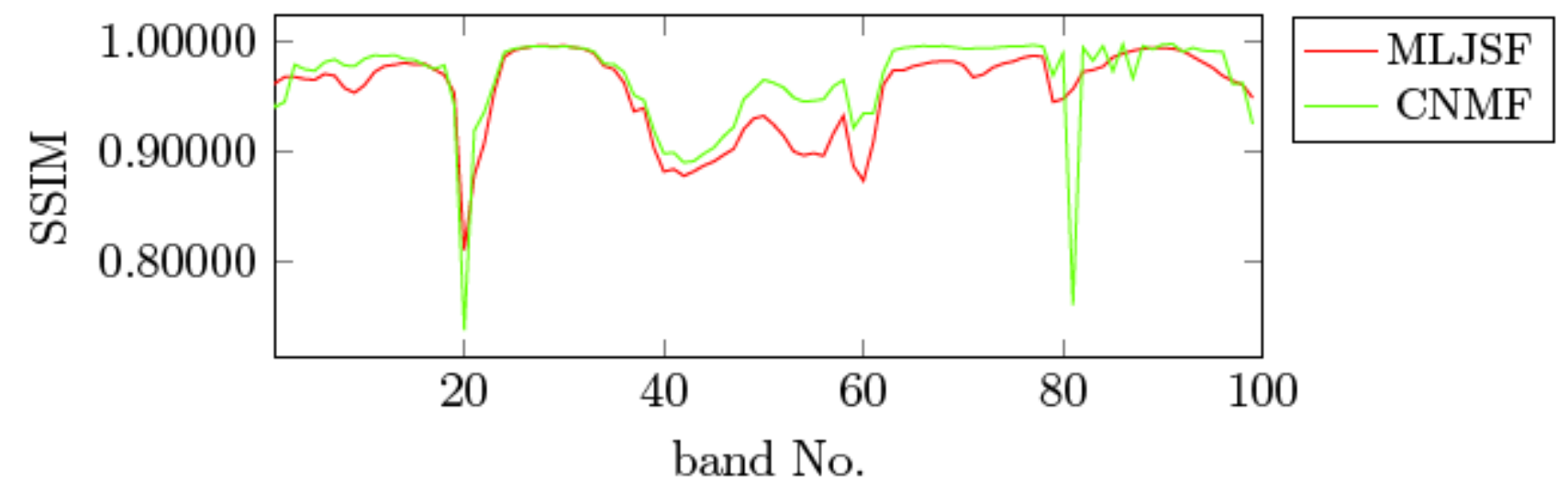




\section{Application to classification \\ $\square$ Road $\square$ Red roof $1 \quad \square$ Red roof $2 \quad \square$ White roof \\ \begin{tabular}{llll} 
Tree & Field 1 \\
\hline Field 2
\end{tabular} \\ $\square$ Field 4 $\square$ Soil 1 $\square$ Soil 2 $\square$ Water}

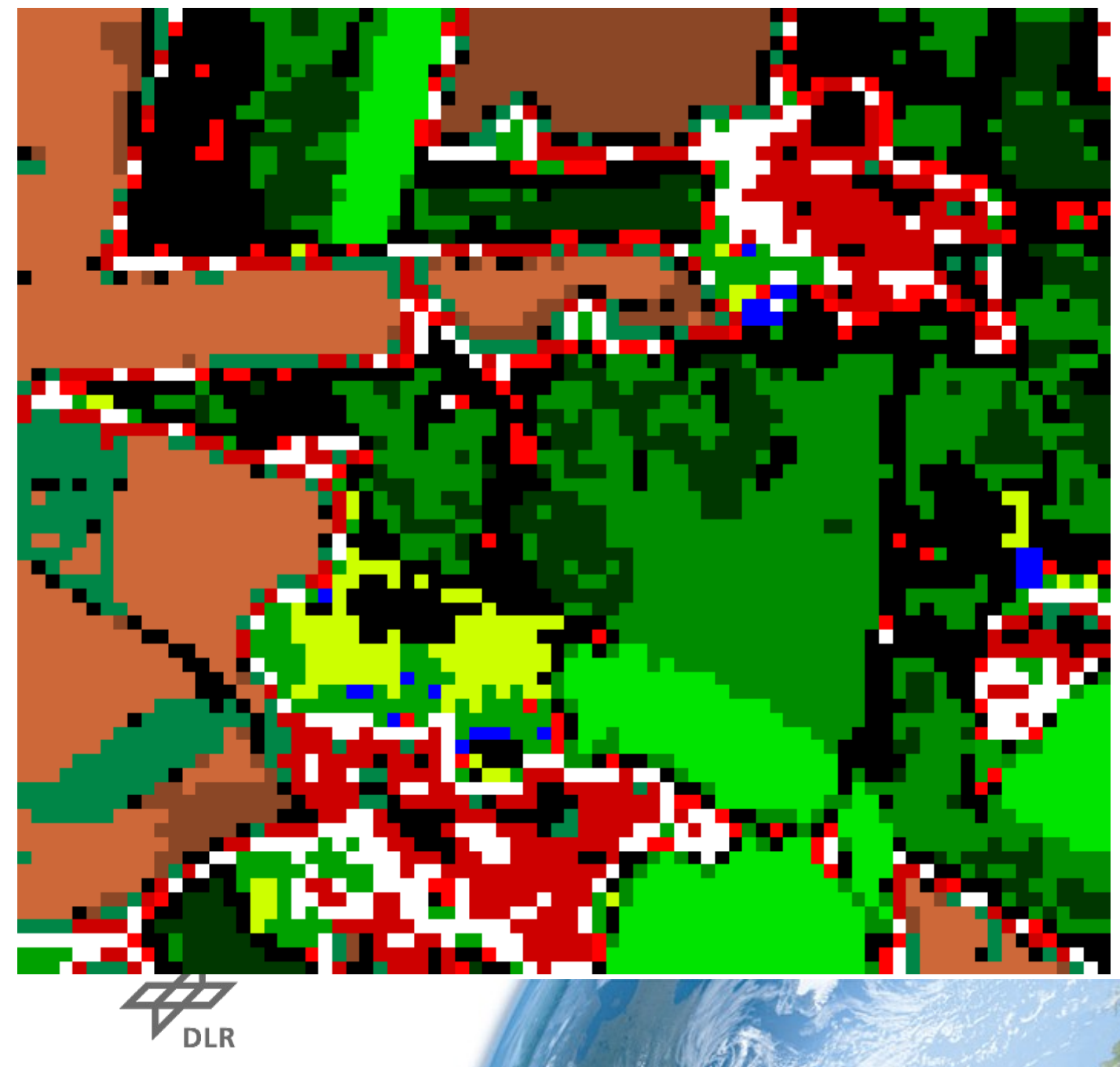

\section{HYPERION}

- 30m resolution

- OA: 42\%

- Kappa:0.36

Compared to classification results of AISA original image 


\section{Application to classification \\ $\square$ Road $\square$ Red roof $1 \square$ Red roof $2 \square$ White roof \\ 口 Tree $\square$ Field 1 $\square$ Field 2 $\square$ Field 3 \\ $\square$ Field 4 $\square$ Soil 1 $\square$ Soil 2 $\square$ Water}

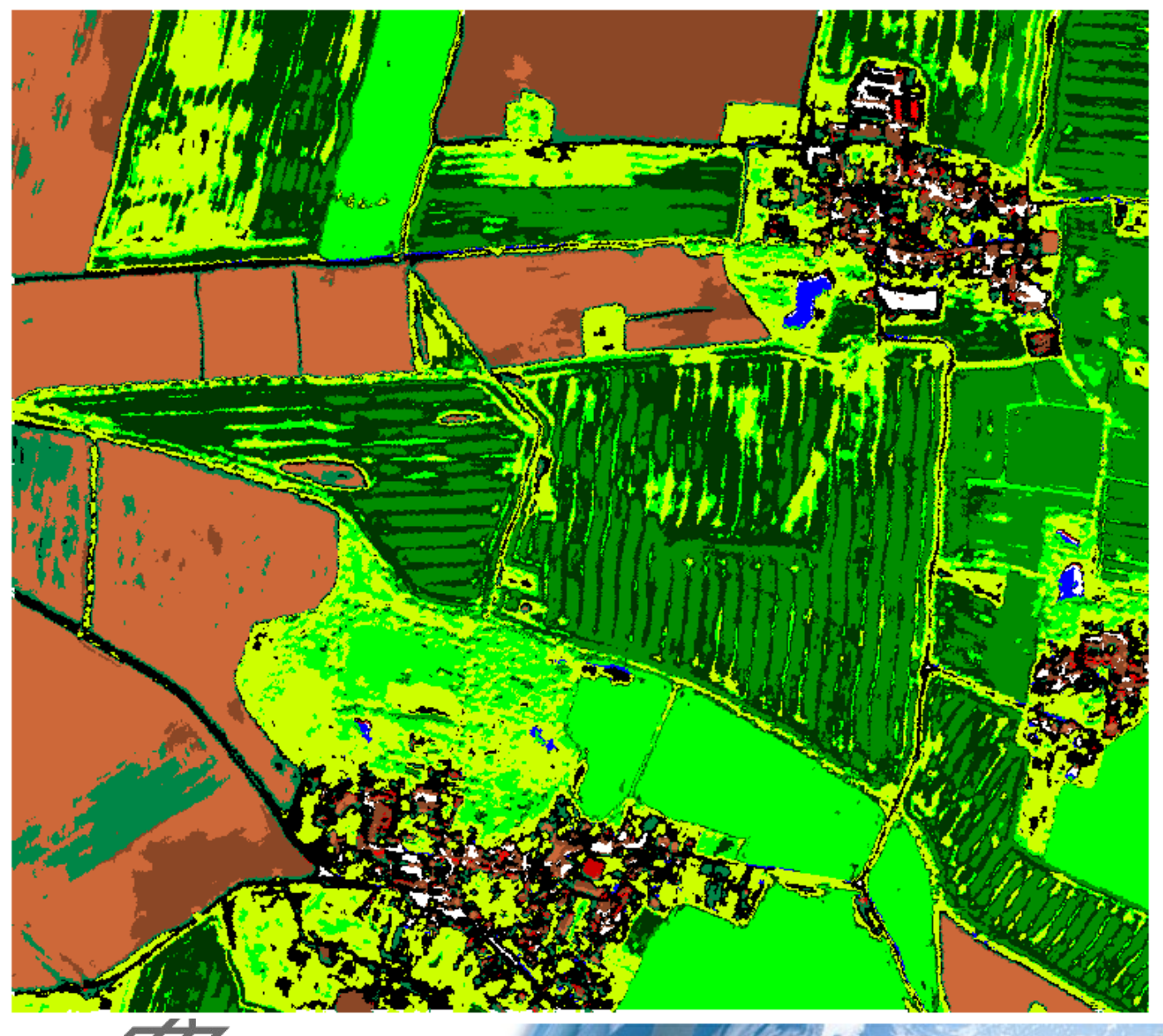

MLJSF

- $3 m$ resolution

- OA: $79 \%$ increase of $37 \%$

- Kappa:0.76 increase of 0.40 


\section{Application to classification \\ $\square$ Road $\square$ Red roof $1 \quad \square$ Red roof $2 \quad \square$ White roof \\ \begin{tabular}{llll} 
Tree & Field 1 \\
\hline Field 2
\end{tabular} \\ $\square$ Field 4 $\square$ Soil 1 $\square$ Soil 2 $\square$ Water}

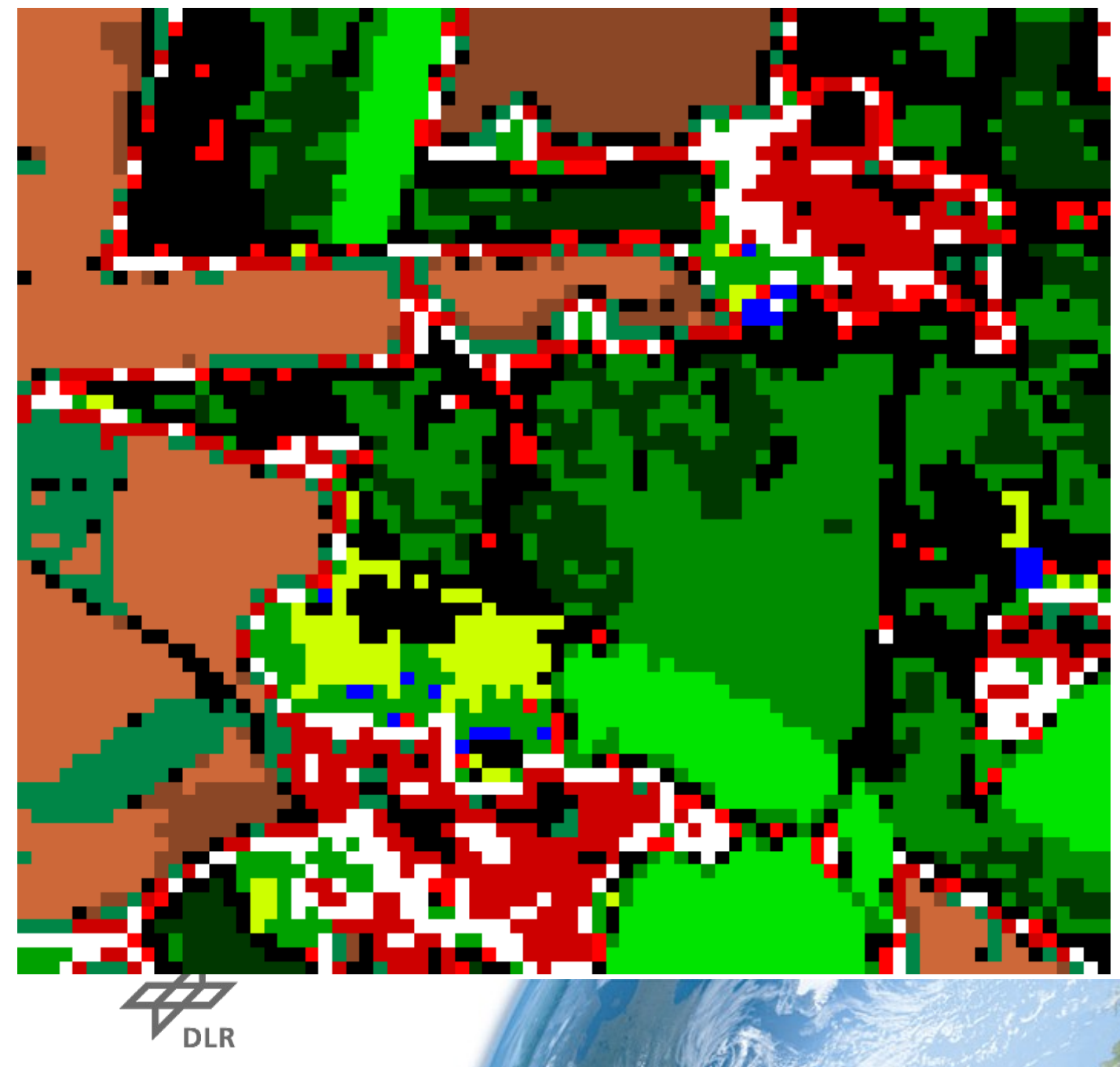

\section{HYPERION}

- 30m resolution

- OA: 42\%

- Kappa:0.36

Compared to classification results of AISA original image 


\section{Application to classification \\ $\square$ Road $\square$ Red roof $1 \quad \square$ Red roof $2 \quad \square$ White roof \\ 口 Tree $\square$ Field 1 $\square$ Field 2 $\square$ Field 3 \\ $\square$ Field 4 $\square$ Soil 1 $\square$ Soil 2 $\square$ Water}

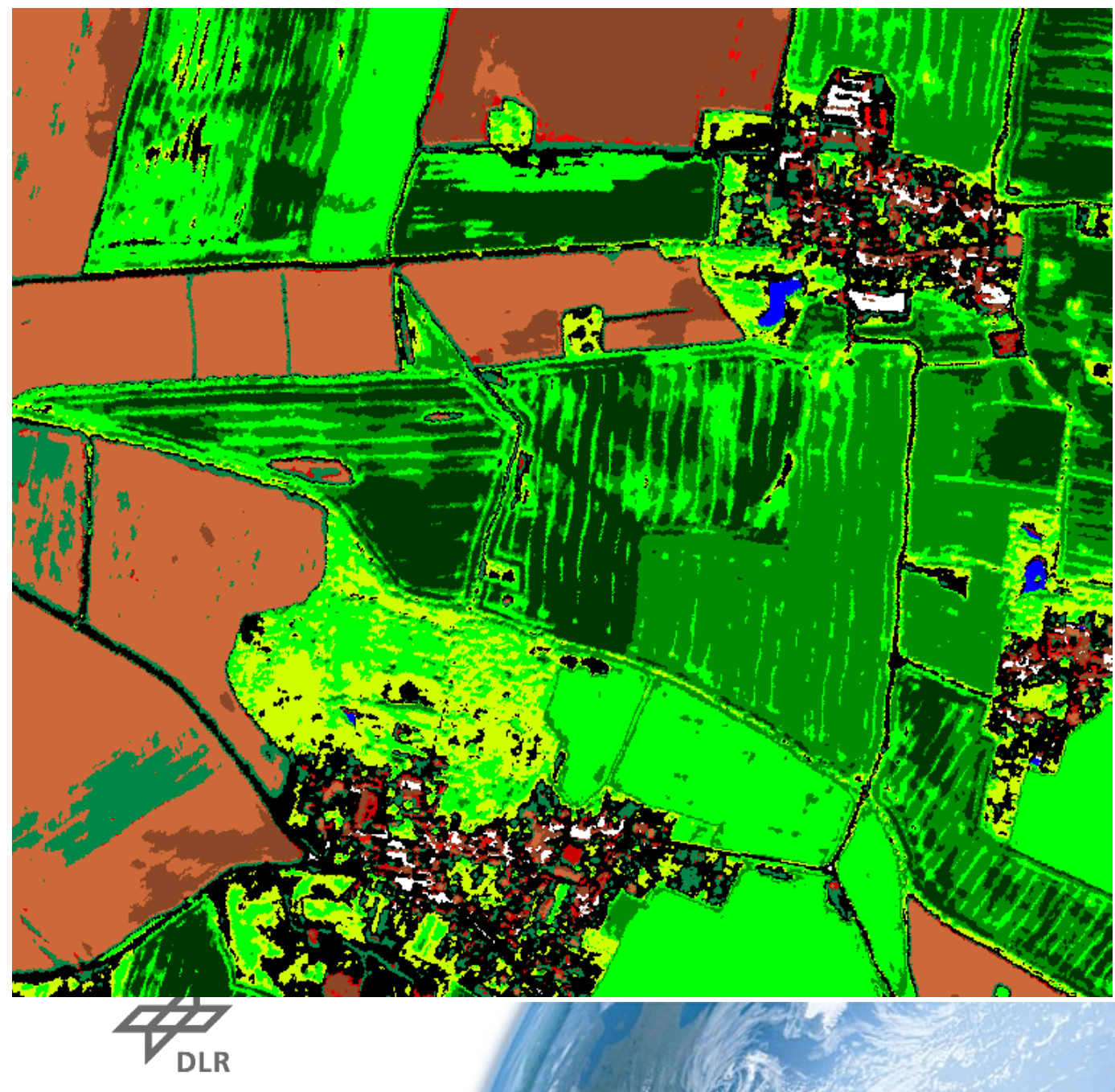

\section{CNMF}

- $3 \mathrm{~m}$ resolution

- OA: 64\% increase of $22 \%$

- Kappa:0.59 increase of 0.23 


\section{Conclusions}

- MLJSF exploits the sparsity in hyperspectral mixtures and the HR spatial information in the multispectral image.

- MLJSF achieves

- Comparable quantitative metrics to the state-of-the-art methods, even when using external libraries containing pure spectra.

- Significantly better performance when aiming at applications like classification.

- Future work: Robustness analysis against coregistration error, different conditions of the data acquisition and missing materials in the library etc. 


\title{
HYPERSPECTRAL RESOLUTION ENHANCEMENT USING MULTISENSOR IMAGE DATA
}

\author{
J. Bieniarz, D. Cerra, X. X. Zhu, R. Müller, P. Reinartz
}

German Aerospace Center (DLR), Remote Sensing Technology Institute (IMF), Oberpfaffenhofen, 82234 Wessling, Germany.

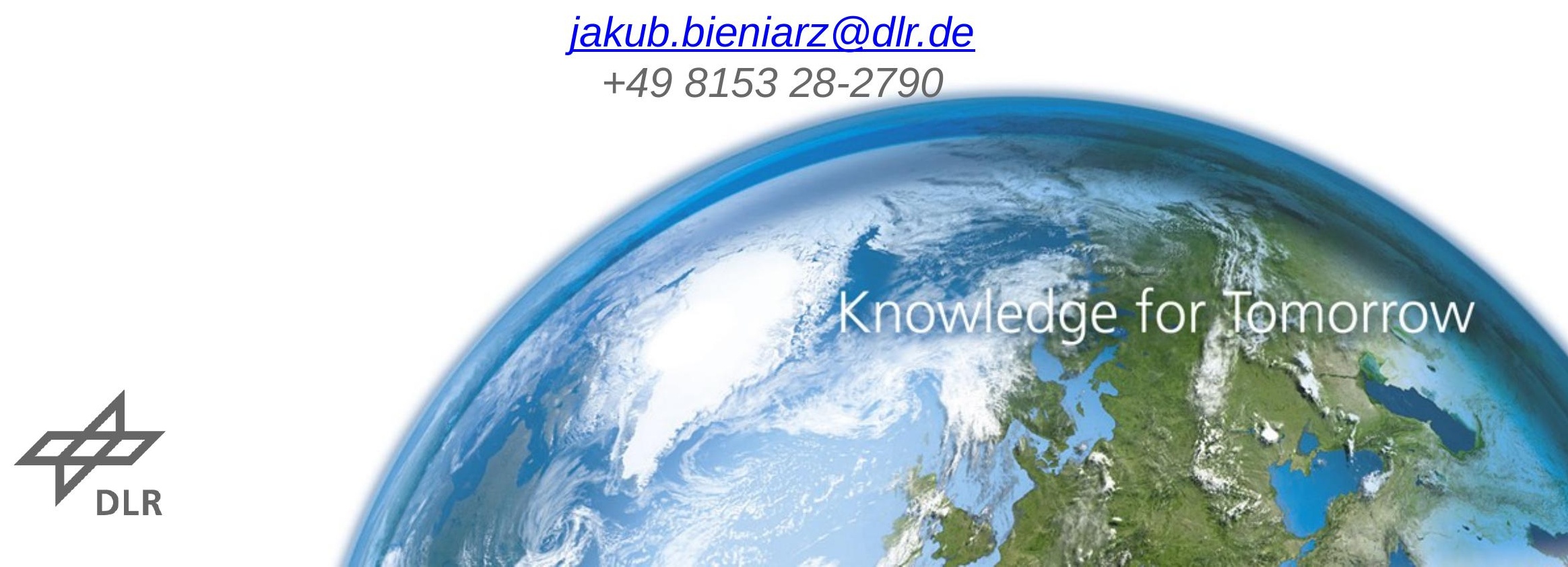




\section{Spectral Resolution}
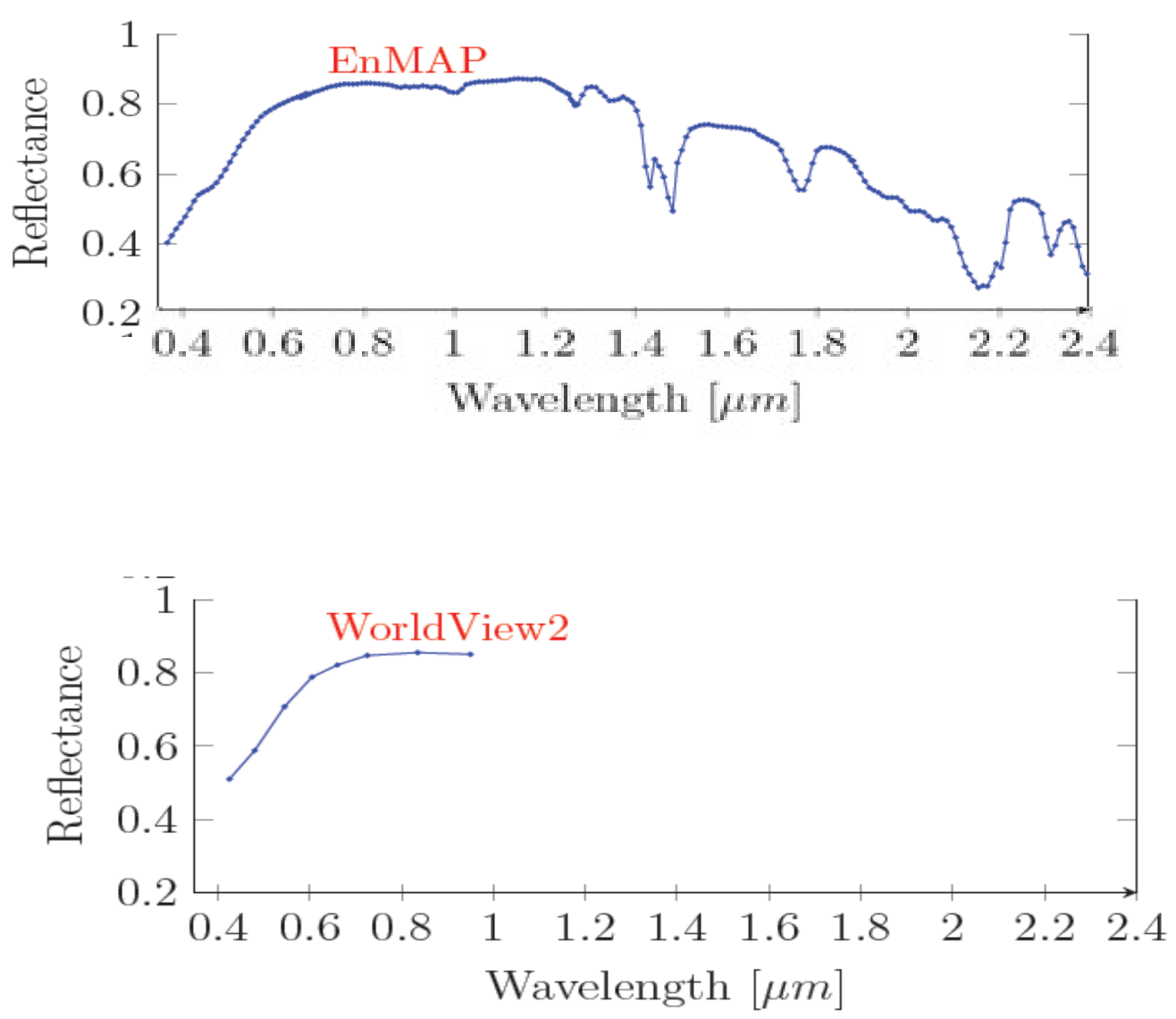


\section{Spectral Resolution}
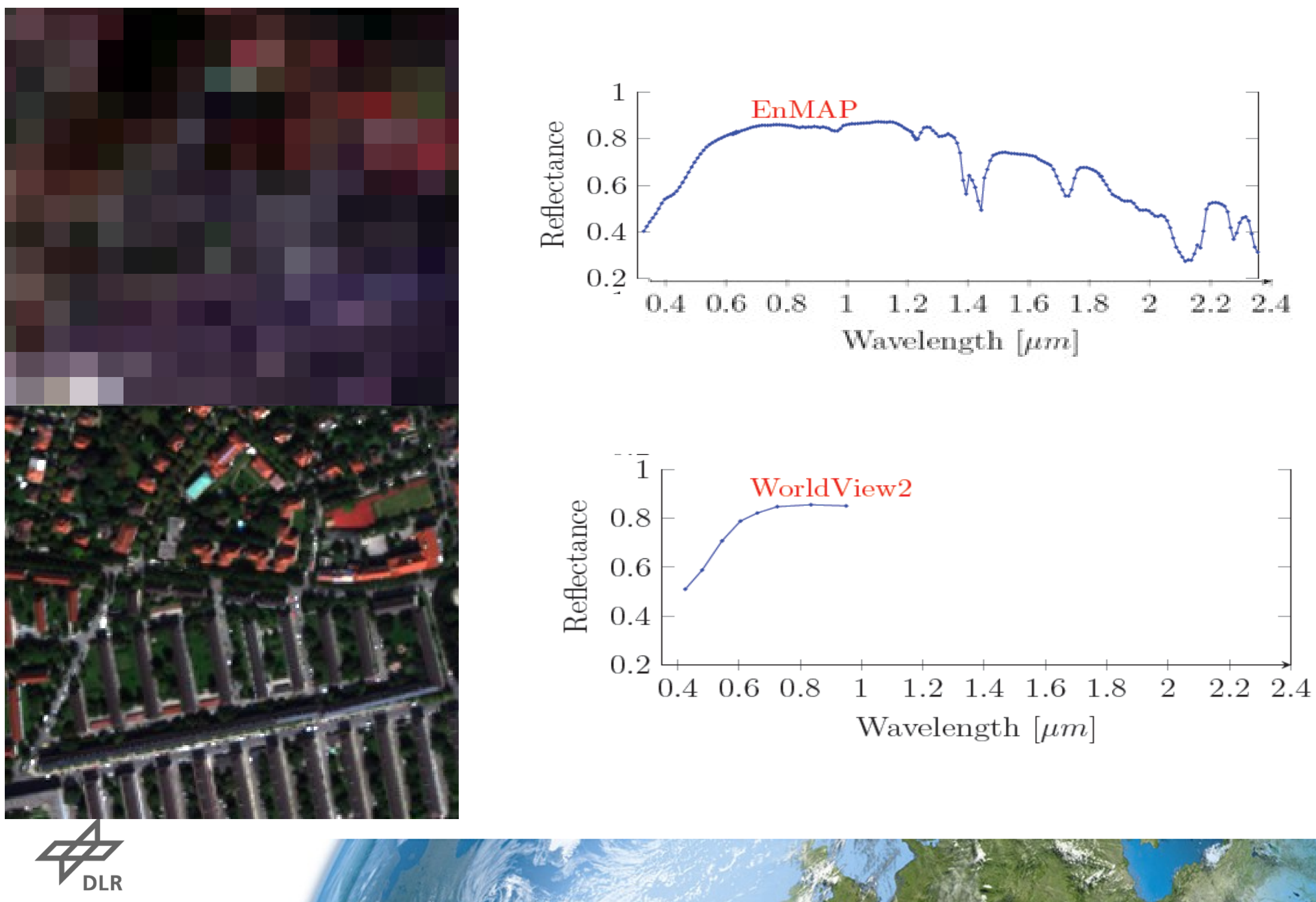


\section{Highly mixed scenario}

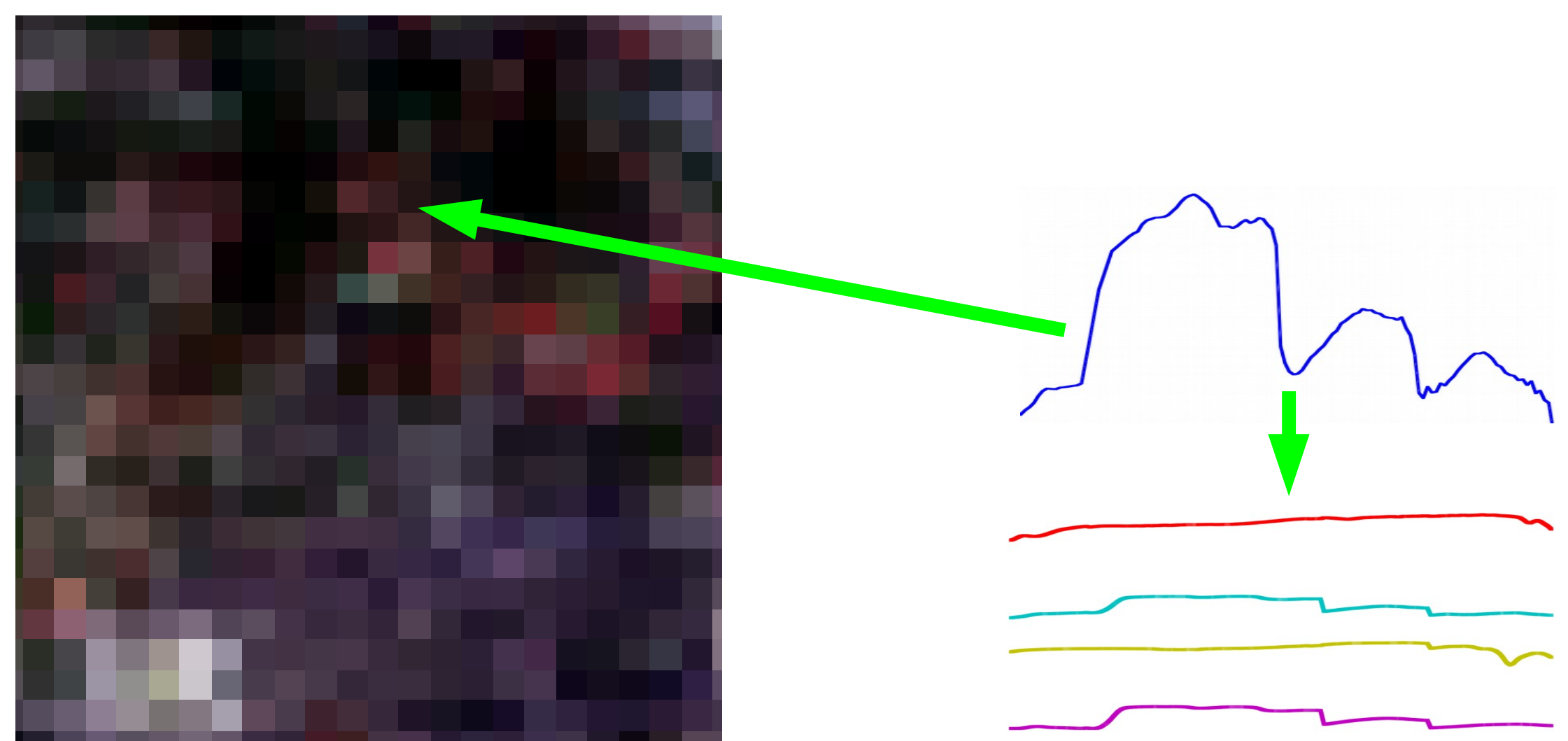




\section{Hyperspectral Image Resolution Enhancement Based on Jointly Sparse Spectral Unmixing}
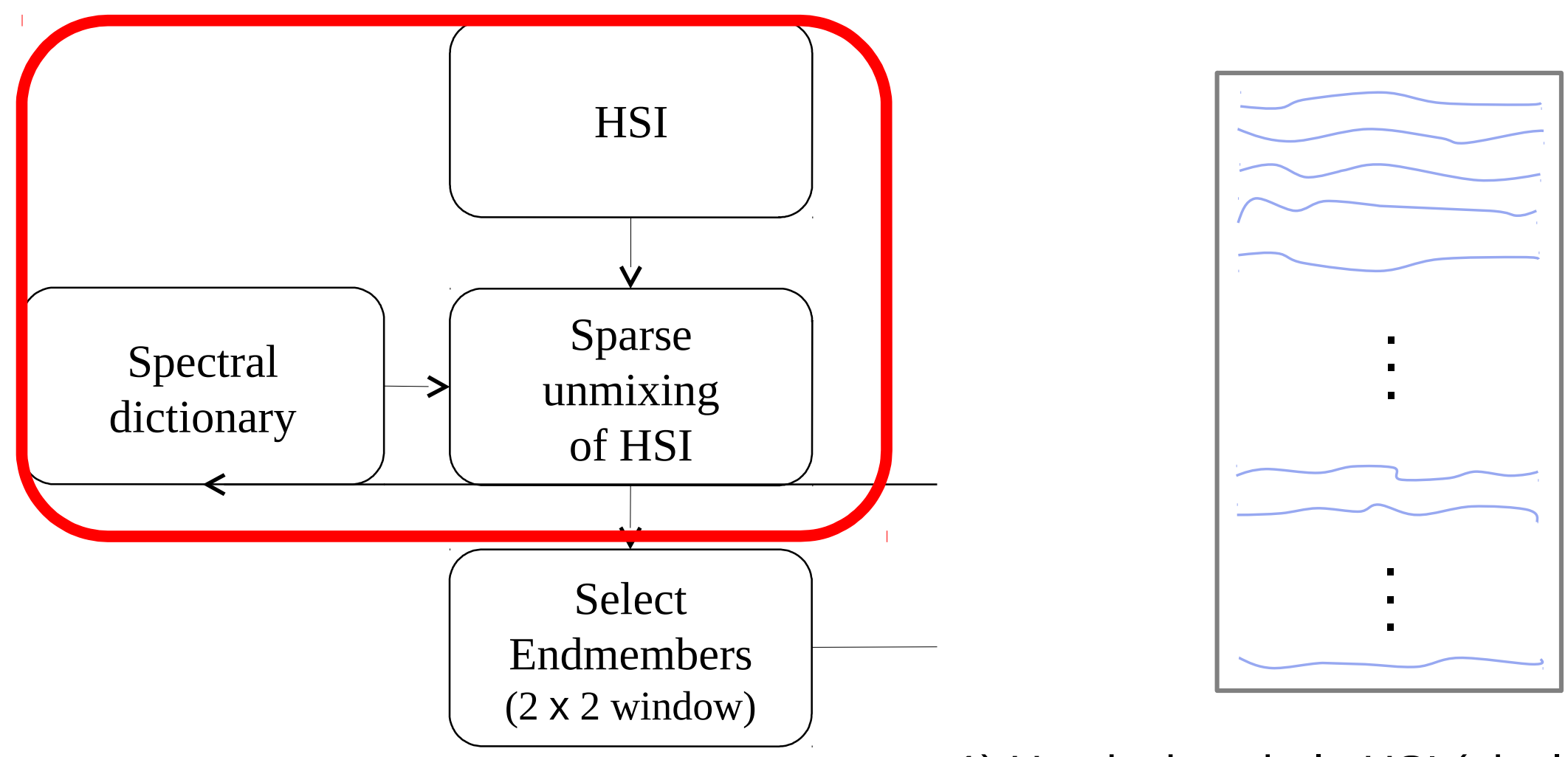

1) Unmix the whole HSI (pixel-wise) using the sparse spectral unmixing method and a priori given spectral dictionary. 


\section{Hyperspectral Image Resolution Enhancement Based on Jointly Sparse Spectral Unmixing}

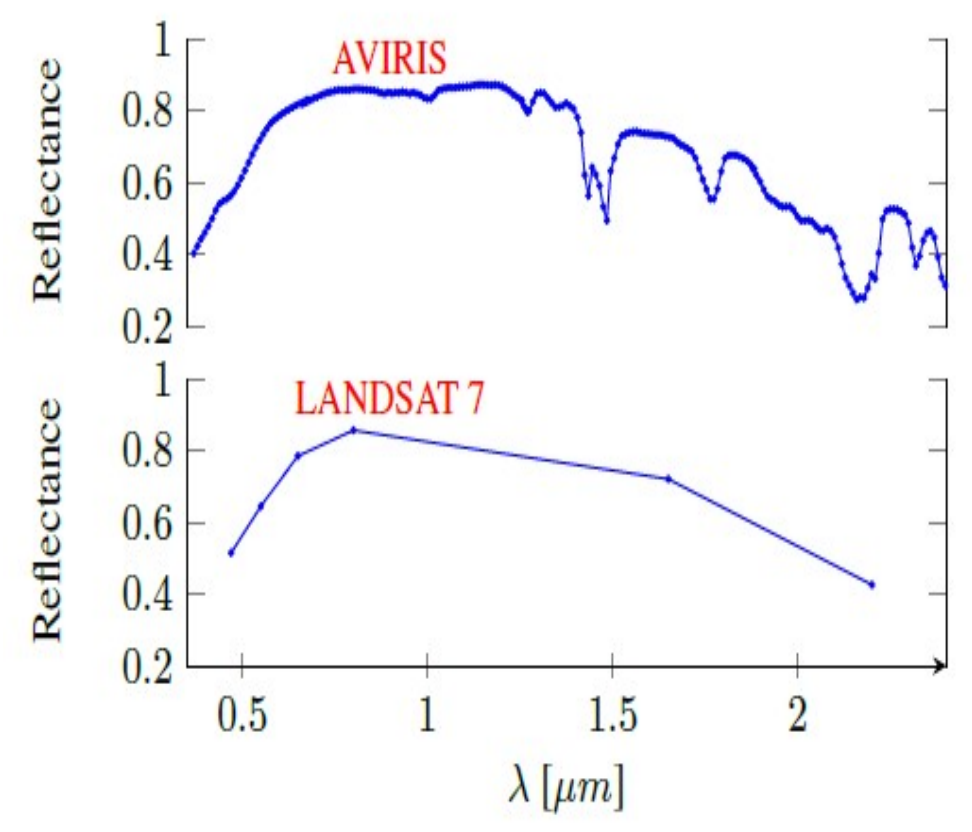

3) Resample endmember spectra to the MSI sensor spectral resolution using the spectral response function (SRF)

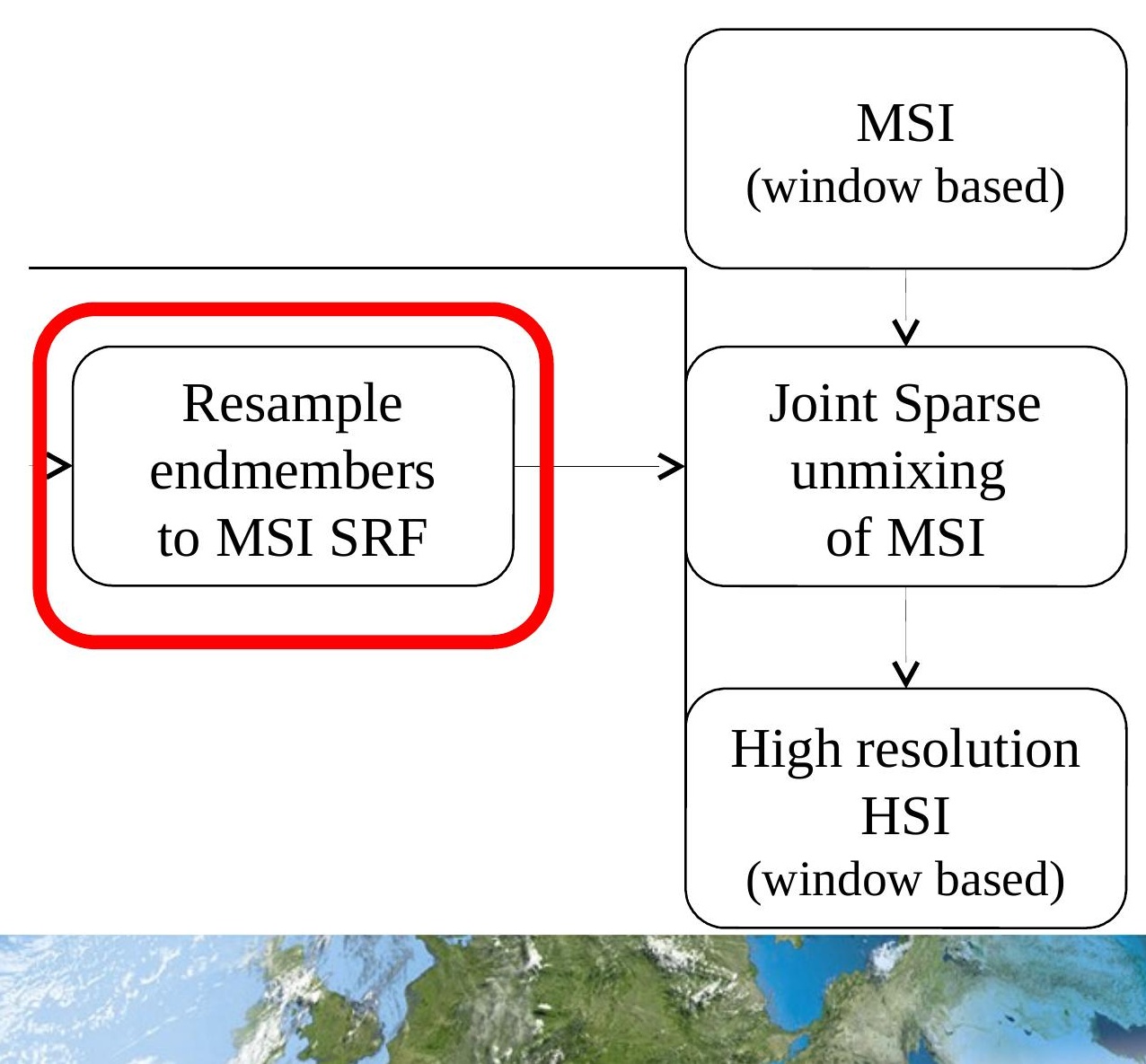




\section{Hyperspectral Image Resolution Enhancement Based on Jointly Sparse Spectral Unmixing}

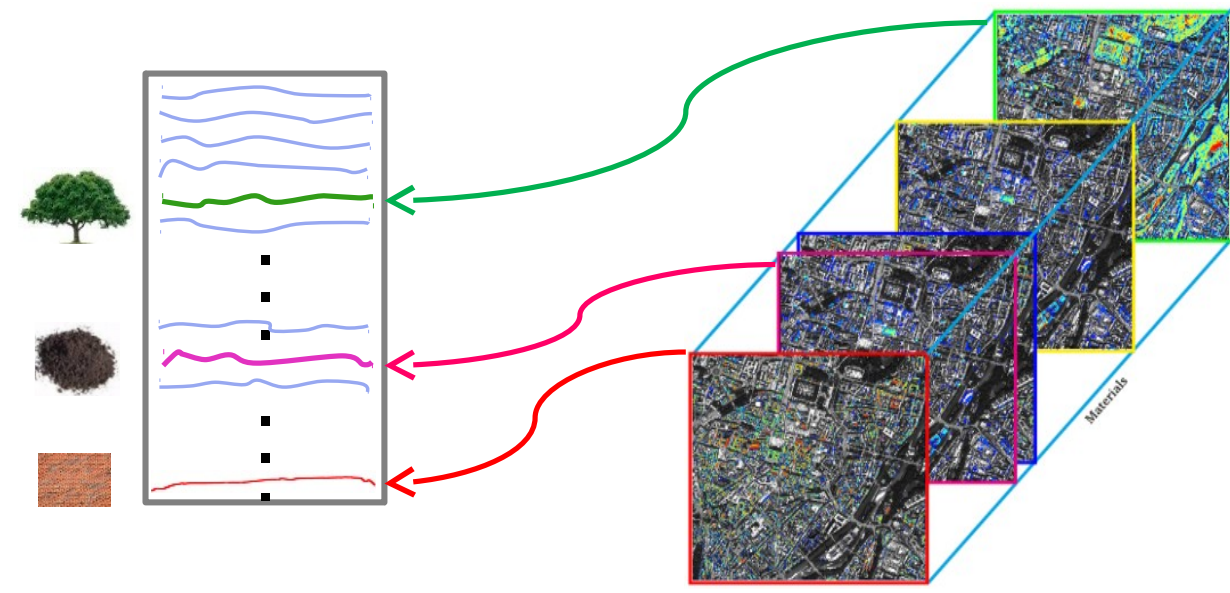

5) reconstruct the high resolution $\mathrm{HSI}$, with

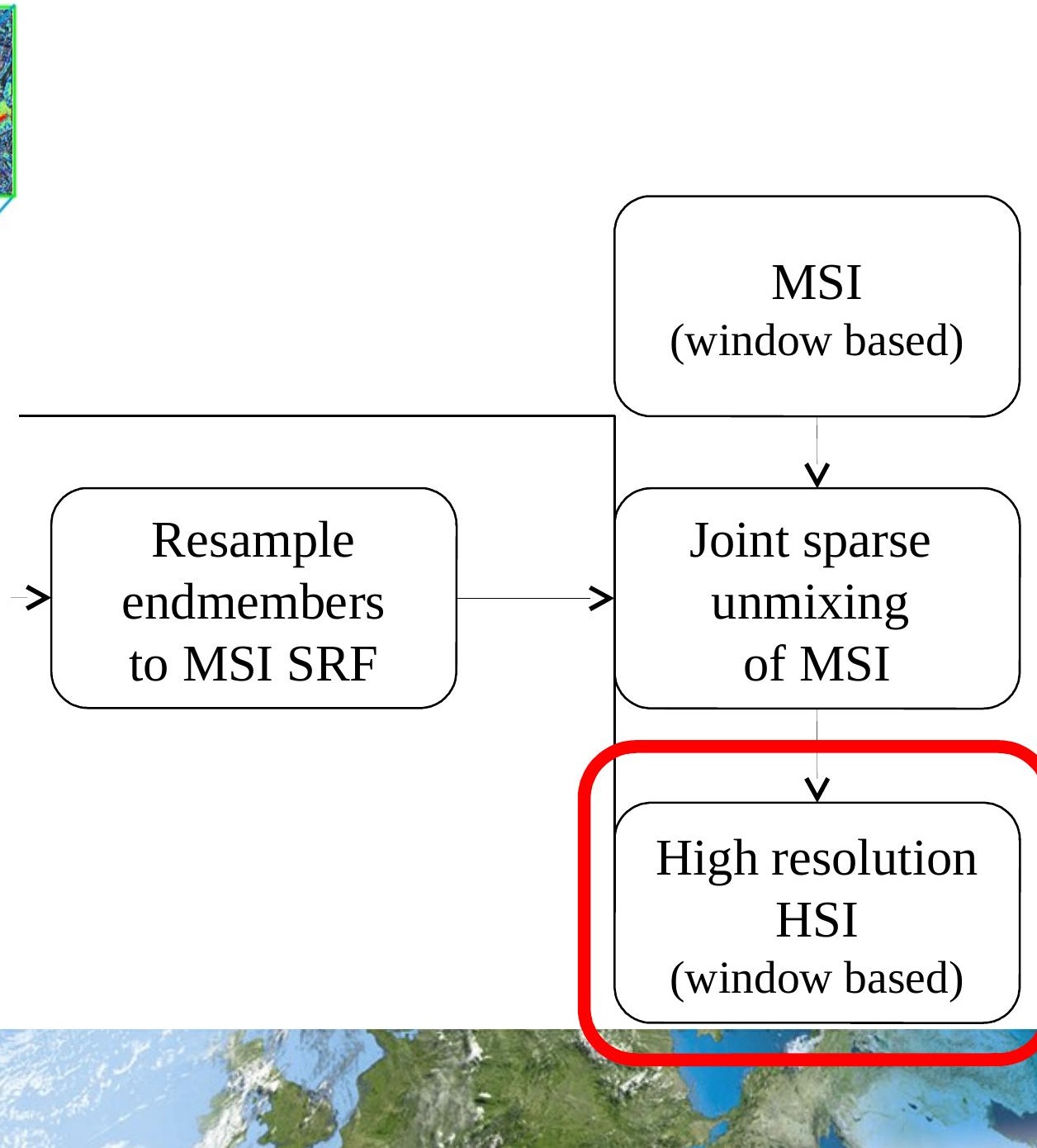
the resulting $\mathrm{MSI}$ abundances and the original HSI spectral dictionary using the LMM. 